\title{
Hardy Spaces on Products of Halfplanes and Prediction of Homogeneous Random Fields
}

\author{
R. Cheng and L. Klotz
}

\begin{abstract}
Using the fractional linear map from the unit disk onto the upper halfplane we transfer some facts about Hardy spaces on polydisks to Hardy spaces on products of halfplanes. The results are applied to the prediction theory of homogeneous random fields on $\mathbb{R}^{n}$. In particular, we give a characterization of regular and weakly (strongly) commutative $L^{2}(\mu)$ spaces.
\end{abstract}

Keywords: Hardy spaces, prediction, random fields

AMS subject classification: 32 A 35, 42 B 30, 60 G 10, 60 G 25, 60 G 60

\section{Introduction}

In this paper we shall use the following notation and conventions. Let $\mathbb{N}, \mathbb{Z}, \mathbb{Z}_{+}, \mathbb{R}$, $\mathbb{R}_{+}$and $\mathbb{C}$ be the sets of natural numbers, integers, non-negative integers, real numbers, non-negative real numbers, and complex numbers, respectively. By $\mathbb{U}, \mathbb{T}$ and $\mathbb{C}_{+}$we denote the open unit disk, the unit circle, and the upper halfplane, respectively. As usual we identify the unit circle $\mathbb{T}$ with the interval $[-\pi, \pi)$ according to the map $[-\pi, \pi) \ni$ $\phi \mapsto e^{i \phi} \in \mathbb{T}$. For $z \in \mathbb{C}$ let $z^{*}$ be the complex conjugate of $z$. A complex number $z$ will have real part $x$ and imaginary part $y$, thus $z=x+i y$. If $z$ is assigned an index or accent, it carries over to $x$ and $y$. By $r$ and $\phi$ we mean the modulus and argument of the complex number $w=r e^{i \phi}$; again, indices and accents carry over. For $n \in \mathbb{N}$ and any set $M$, we write $M^{n}$ for the $n$-fold Cartesian product of $M$. Let $\lambda_{n}$ and $\sigma_{n}$ be the Lebesgue measure on $\mathbb{R}^{n}$ and $\mathbb{T}^{n}$, respectively. If the quantifiers a.a. (almost all) or a.e. (almost everywhere) are used without specifying a measure, then the appropriate Lebesgue measure is assumed. A non-negative measure we simply call a measure; otherwise, we speak of a real-or a complex-valued measure. We shall consider the Banach spaces $L^{P}(\mu)$, where $\mu$ is a measure and $1 \leq p \leq \infty$. The closure of a subset $M$ of a topological space is written $\bar{M}$. The closed linear span of a subset $M$ of $L^{p}(\mu)$ is written $\vee_{p, \mu} M$; when no measure is specified we assume Lebesgue measure.

For $p \in[1, \infty)$ and $n \in \mathbb{N}$, we examine the following function spaces:

R. Cheng: Univ. Louisville, Dept. Math., Louisville, KY 40292, U.S.A.

L. Klotz: Univ. Leipzig, Inst. Math., Augustuspl. 10 - 11, D - 04109 Leipzig

ISSN 0232-2064 / \$2.50 c Heldermann Verlag Berlin 
- $H^{p}\left(\mathbb{C}_{+}^{n}\right)$ consists of functions $f$ analytic on $\mathbb{C}_{+}^{n}$ and such that

$$
\sup _{\left(y_{1}, \ldots, y_{n}\right) \in(0, \infty)^{n}} \int_{\mathbb{R}^{n}}\left|f\left(x_{1}+i y_{1}, \ldots, x_{n}+i y_{n}\right)\right|^{p} \lambda_{n}\left(d x_{1} \times \cdots \times d x_{n}\right)<\infty .
$$

- $H^{p}\left(\mathbb{U}^{n}\right)$ consists of functions $f$ analytic on $\mathbb{U}^{n}$ and such that

$$
\sup _{\left(r_{1}, \ldots, r_{n}\right) \in(0,1)^{n}} \int_{(-\pi, \pi)^{n}}\left|f\left(r_{1} e^{i \phi_{1}}, \ldots, r_{n} e^{i \phi_{n}}\right)\right|^{p} \sigma_{n}\left(d \phi_{1} \times \cdots \times d \phi_{n}\right)<\infty .
$$

- $\mathcal{H}^{p}\left(\mathbb{C}_{+}^{n}\right)$ consists of functions $f$ analytic on $\mathbb{C}_{+}^{n}$ and such that

$$
\sup _{\left(y_{1}, \ldots, y_{n}\right) \in(0, \infty)^{n}} \int_{\mathbb{R}^{n}} \frac{\left|f\left(x_{1}+i y_{1}, \ldots, x_{n}+i y_{n}\right)\right|^{p}}{\left(x_{1}^{2}+\left[y_{1}+1\right]^{2}\right) \cdots\left(x_{n}^{2}+\left[y_{n}+1\right]^{2}\right)} \lambda_{n}\left(d x_{1} \times \cdots \times d x_{n}\right)<\infty .
$$

Moreover:

- $H^{\infty}\left(\mathbb{C}_{+}^{n}\right):=\mathcal{H}^{\infty}\left(\mathbb{C}_{+}^{n}\right)$ consists of functions $f$ analytic on $\mathbb{C}_{+}^{n}$ and such that

$$
\sup _{\left(z_{1}, \ldots, z_{n}\right) \in \mathbf{C}_{+}^{n}}\left|f\left(z_{1}, \ldots, z_{n}\right)\right|<\infty
$$

- $H^{\infty}\left(\mathbb{U}^{n}\right)$ consists of functions $f$ analytic on $\mathbb{U}^{n}$ and such that

$$
\left.\sup _{\left(w_{1}, \ldots, w_{n}\right) \in \mathbb{U}^{n}}\left|f\left(w_{1}, \ldots, w_{n}\right)\right|<\infty\right\}
$$

It is obvious that $H^{p}\left(\mathbb{C}_{+}^{n}\right) \subseteq \mathcal{H}^{p}\left(\mathbb{C}_{+}^{n}\right)$

The spaces $H^{p}\left(\mathbb{C}_{+}\right)$and $H^{p}(\mathbb{U})$ are the classical Hardy spaces on the upper halfplane and unit disk, respectively. For over three decades there have been efforts to generalize the results from functions of one variable to functions of several variables. Rudin's book [15] was the first unifying exposition of the theory for $H^{p}\left(\mathbb{U}^{n}\right)$. An important application of the $H^{2}\left(\mathbb{U}^{n}\right)$ case is in linear prediction problems for homogeneous random fields on $\mathbb{Z}^{n}$. In the present paper we wish to develop the theory of $H^{p}\left(\mathbb{C}_{+}^{n}\right)$ spaces, and to apply these results to prediction problems for homogeneous random fields on $\mathbb{R}^{n}$. For the sake of notational simplicity, we take $n=2$.

Many properties of $H^{p}\left(\mathbb{C}_{+}^{2}\right)$ functions can be derived from the corresponding properties of $H^{p}(\mathbb{U})$ functions with the aid of a fractional linear map $\alpha$ from $\mathbb{U}$ onto $\mathbb{C}_{+}$. In using this method the spaces $\mathcal{H}^{p}\left(\mathbb{C}_{+}^{2}\right)$ occur in a natural way, as $\alpha$ induces a one-toone linear correspondence from $H^{p}\left(\mathbb{U}^{2}\right)$ onto $\mathcal{H}^{p}\left(\mathbb{C}_{+}^{2}\right)$. This fact rests on some results concerning 2-harmonic and 2-subharmonic functions, particularly a theorem of Flett and Kuran (extended to several variables), contained in Section 1. The proof of the several-variables version of the theorem of Flett and Kuran is obtained by a modification of one-variable arguments as given in [14: pp. $136-139]$. Section 2 is devoted to basic properties of $H^{p}\left(\mathbb{C}_{+}^{2}\right)$ functions; the methods and outcomes are generally such as one would expect. The key property is the behavior of non-tangential limits. We derive it from Zygmund's results on $H^{p}\left(\mathbb{U}^{2}\right)$ functions (cf. [17]), with the aid of the 
map $\alpha$. Then other facts as Cauchy's formula and embedding in $L^{p}\left(\lambda_{2}\right)$ can be proved. In Section 4 we study various notions of outer functions in $H^{P}\left(\mathbb{C}_{+}^{2}\right)$. We obtain an explicit correspondence between the weakly outer, outer, and strongly outer functions in $H^{p}\left(\mathbb{U}^{2}\right)$ and their counterparts in $H^{p}\left(\mathbb{C}_{+}^{2}\right)$. The results concerning weakly outer and outer functions are easy to derive, whereas the proof of the correspondence between strongly outer functions uses some approximation properties in $L^{P}(\mu)$ spaces of functions of several variables, which we obtain in Section 3 applying techniques from [1]. The assertions of Section 4 combined with Theorem 1.1 in [2] yield a characterization of regular and weakly (strongly) commutative $L^{2}(\mu)$ spaces. This and some other results, which are of interest in the prediction theory of homogeneous random fields on $\mathbb{R}^{n}$, are given in Section 5 .

\section{Non-negative 2-subharmonic functions on $\mathbb{C}_{+}^{2}$}

Consider the fractional linear mapping $\alpha: \overline{\mathbb{U}} \mapsto \overline{\mathbb{C}}_{+} \cup\{\infty\}$ given by

$$
\alpha(w)=i \frac{1-w}{1+w}
$$

Its inverse is $\beta$, where

$$
\beta(z)=\frac{i-z}{i+z} .
$$

Restrictions of $\alpha$ and $\beta$ to subsets of their domains are also denoted by $\alpha$ and $\beta$, respectively. If $f$ is a function on a subset $M \times N$ of $\overline{\mathbb{C}_{+}^{2}}$, we define

$$
f_{\alpha}\left(w_{1}, w_{2}\right)=f\left(\alpha\left(w_{1}\right), \alpha\left(w_{2}\right)\right)
$$

for $\left(w_{1}, w_{2}\right) \in \beta(M) \times \beta(N)$. Similarly, if $g$ is a function on a subset $M \times N$ of $\overline{\mathbb{U}^{2}}$, we define

$$
g_{\beta}\left(z_{1}, z_{2}\right)=g\left(\beta\left(z_{1}\right), \beta\left(z_{2}\right)\right)
$$

for $\left(z_{1}, z_{2}\right) \in \alpha(M) \times \alpha(N)$. Thus these two mappings will serve to bridge the well established theory on $\mathbb{U}^{2}$ and the situation on $\mathbb{C}_{+}^{2}$.

A function defined on an open subset of $\mathbb{C}^{2}$ is 2-harmonic or 2-subharmonic, if it is harmonic or subharmonic, respectively, in each variable [15: Subsections 2.1.1 and 3.2.1]. We shall establish a Poisson integral representation for 2-harmonic functions on $\mathbb{C}_{+}^{2}$, as well as a criterion for 2-harmonic majorants for certain functions on $\mathbb{C}_{+}^{2}$.

By $P$ we denote the Poisson kernel on $\mathbb{C}_{+}$, thus

$$
P(x, y)=\frac{1}{\pi} \frac{y}{x^{2}+y^{2}}
$$

for $z=x+i y \in \mathbb{C}_{+}$. The Poisson kernel on $\mathbb{U}$ is given by

$$
P^{\circ}(\phi, r)=\frac{1}{2 \pi} \frac{1-r^{2}}{1-2 r \cos \phi+r^{2}}
$$


for $w=r e^{i \phi} \in \mathbb{U}$. If $\mu$ and $\nu$ are bounded complex-valued Borel measures on $\mathbb{R}^{2}$ and $\mathbb{T}^{2}$, respectively, we express their Poisson integrals in the following way:

$$
P[d \mu]\left(z_{1}, z_{2}\right)=\int P\left(x_{1}-t_{1}, y_{1}\right) P\left(x_{2}-t_{2}, y_{2}\right) \mu\left(d t_{1} \times d t_{2}\right)
$$

for $\left(z_{1}, z_{2}\right)=\left(x_{1}+i y_{1}, x_{2}+i y_{2}\right) \in \mathbb{C}_{+}^{2}$, and

$$
P^{\circ}[d \nu]\left(w_{1}, w_{2}\right)=\int P^{\circ}\left(\phi_{1}-\theta_{1}, r_{1}\right) P^{\circ}\left(\phi_{2}-\theta_{2}, r_{2}\right) \nu\left(d \theta_{1} \times \theta_{2}\right)
$$

for $\left(w_{1}, w_{2}\right)=\left(r_{1} e^{i \phi_{1}}, r_{2} e^{i \phi_{2}}\right) \in \mathbb{U}^{2}$. If $\mu$ is of the form $d \mu=f d \lambda_{2}$ for some $f \in L^{1}\left(\lambda_{2}\right)$, then we write $P[f]$ instead of $P[\mu]$; a similar convention applies to $P^{\circ}$.

Lemma 1.1. A function $h$ defined on $\mathbb{C}_{+}^{2}$ is non-negative and 2-harmonic if and only if it has the representation

$$
h\left(z_{1}, z_{2}\right)=P[d \mu]\left(z_{1}, z_{2}\right)+y_{1} P\left[d \mu_{2}\right]\left(z_{2}\right)+y_{2} P\left[d \mu_{1}\right]\left(z_{1}\right)+\rho y_{1} y_{2}
$$

where $\rho \in \mathbb{R}_{+}, \mu$ is a Borel measure on $\mathbb{R}^{2}, \mu_{1}$ and $\mu_{2}$ are Borel measures on $\mathbb{R}$, and

$$
\begin{array}{r}
\int_{\mathbb{R}^{2}}\left(1+t_{1}^{2}\right)^{-1}\left(1+t_{2}^{2}\right)^{-1} \mu\left(d t_{1} \times d t_{2}\right)<\infty \\
\int_{\mathbb{R}}\left(1+t^{2}\right)^{-1} \mu_{1}(d t)<\infty \\
\int_{\mathbb{R}}\left(1+t^{2}\right)^{-1} \mu_{2}(d t)<\infty .
\end{array}
$$

Proof. It is obvious that a function $h$ having the representation (1) is non-negative and 2-harmonic. Now let $h$ be a non-negative and 2-harmonic function on $\mathbb{C}_{+}^{2}$. Then $h_{\alpha}$ is non-negative and 2-harmonic on $\mathbb{U}^{2}$, and there exists a Borel measure $\nu$ on $\mathbb{T}^{2}$ such that

$$
h_{\alpha}\left(w_{1}, w_{2}\right)=P^{\circ}[d \nu]\left(w_{1}, w_{2}\right)
$$

for $\left(w_{1}, w_{2}\right) \in \mathbb{U}^{2}$. Write the integral on the right side of $(2)$ as a sum of four integrals over the sets $(-\pi, \pi)^{2},\{-\pi\} \times(-\pi, \pi),(-\pi, \pi) \times\{-\pi\}$ and $\{-\pi\}^{2}$. Define a Borel measure $\mu$ on $\mathbb{R}^{2}$ by

$$
\mu\left(d t_{1} \times d t_{2}\right)=\left(1+t_{1}^{2}\right)\left(1+t_{2}^{2}\right) \nu\left(\beta\left(d t_{1}\right) \times \beta\left(d t_{2}\right)\right) .
$$

We have

$$
\int_{\mathbb{R}^{2}}\left(1+t_{1}^{2}\right)^{-1}\left(1+t_{2}^{2}\right)^{-1} \mu\left(d t_{1} \times d t_{2}\right)<\infty .
$$

If we use the relation $r_{j} e^{i \phi_{j}}=\frac{i-z_{j}}{i+z_{j}}(j=1,2)$, a calculation analogous to that in [11: Subsection VI.A] shows that

$$
\int_{(-\pi, \pi)^{2}} P^{\circ}\left(\phi_{1}-\theta_{1}, r_{1}\right) P^{\circ}\left(\phi_{2}-\theta_{2}, r_{2}\right) \nu\left(d \theta_{1} \times d \theta_{2}\right)=P[d \mu]\left(z_{1}, z_{2}\right)
$$


for $\left(z_{1}, z_{2}\right) \in \mathbb{C}_{+}^{2}$. Furthermore, it holds that

$$
\begin{array}{r}
\int_{\{-\pi\} \times(-\pi, \pi)} P^{\circ}\left(\phi_{1}-\theta_{1}, r_{1}\right) P^{\circ}\left(\phi_{2}-\theta_{2}, r_{2}\right) \nu\left(d \theta_{1} \times d \theta_{2}\right) \\
=P^{\circ}\left(\phi_{1}+\pi, r_{1}\right) \int_{(-\pi, \pi)} P^{\circ}\left(\phi_{2}-\theta_{2}, r_{2}\right) \nu\left(\{-\pi\} \times d \theta_{2}\right)
\end{array}
$$

The Borel measure $\mu_{2}$ on $\mathbb{R}$ given by $\mu_{2}\left(d t_{2}\right)=\left(1+t_{2}^{2}\right) \nu\left(\{-\pi\} \times \beta\left(d t_{2}\right)\right)$ has the property $\int_{\mathbb{R}}\left(1+t^{2}\right)^{-1} \mu_{2}(d t)<\infty$, and as before we obtain

$$
\int_{(-\pi, \pi)} P^{\circ}\left(\phi_{2}-\theta_{2}, r_{2}\right) \nu\left(\{-\pi\} \times d \theta_{2}\right)=P\left[d \mu_{2}\right]\left(z_{2}\right)
$$

for $z_{2} \in \mathbb{C}$. Using $r_{1} e^{i \phi_{1}}=\frac{i-z_{1}}{i+z_{1}}$ we get $P^{\circ}\left(\phi_{1}+\pi, r_{1}\right)=\frac{y_{1}}{\pi}$. Hence

$$
\int_{\{-\pi\} \times(-\pi, \pi)} P^{\circ}\left(\phi_{1}-\theta_{1}, r_{1}\right) P^{\circ}\left(\phi_{2}-\theta_{2}, r_{2}\right) \nu\left(d \theta_{1} \times d \theta_{2}\right)=\frac{y_{1}}{\pi} P\left[d \mu_{2}\right]\left(z_{2}\right)
$$

for $\left(z_{1}, z_{2}\right) \in \mathbb{C}_{+}^{2}$. In an analogous fashion we obtain a measure $\mu_{1}$ satisfying

$$
\int_{(-\pi, \pi) \times\{-\pi\}} P^{\circ}\left(\phi_{1}-\theta_{1}, r_{1}\right) P^{\circ}\left(\phi_{2}-\theta_{2}, r_{2}\right) \nu\left(d \theta_{1} \times d \theta_{2}\right)=\frac{y_{2}}{\pi} P\left[d \mu_{1}\right]\left(z_{1}\right)
$$

for $\left(z_{1}, z_{2}\right) \in \mathbb{C}_{+}^{2}$. Finally we calculate

$$
\begin{aligned}
\int_{\{-\pi\}^{2}} P^{\circ}\left(\phi_{1}-\theta_{1}, r_{1}\right) P^{\circ}\left(\phi_{2}-\theta_{2}, r_{2}\right) \nu\left(d \theta_{1} \times d \theta_{2}\right) \\
=P^{\circ}\left(\phi_{1}+\pi, r_{1}\right) P^{\circ}\left(\phi_{2}+\pi, r_{2}\right) \nu\left(\{-\pi\}^{2}\right) \\
=\rho y_{1} y_{2}
\end{aligned}
$$

where $\rho=\frac{1}{\pi^{2}} \nu\left(\{-\pi\}^{2}\right) \geq 0$. This confirms the representation (2)

Now we generalize a theorem of Flett and Kuran on non-negative subharmonic functions on $\mathbb{C}_{+}$to non-negative 2 -subharmonic functions on $\mathbb{C}_{+}^{2}$. This makes it possible to characterize the elements of $\mathcal{H}^{p}\left(\mathbb{C}_{+}^{2}\right)$, and eventually to obtain an explicit one-toone correspondence between $H^{p}\left(\mathbb{U}^{2}\right)$ and $H^{p}\left(\mathbb{C}_{+}^{2}\right)$. Our methods derive from the onevariable proofs in [14].

Let us adopt the following notation. For $s_{1}$ and $s_{2}$ in $\mathbb{R}$, let $u_{s_{1}, s_{2}}$ be the function defined by

$$
u_{s_{1}, s_{2}}\left(z_{1}, z_{2}\right)=\left(z_{1}+i\right)^{-s_{1}}\left(z_{2}+i\right)^{-s_{2}}
$$

for $\left(z_{1}, z_{2}\right) \in \overline{\mathbb{C}_{+}^{2}}$, where the analytic branches satisfying $i^{-s}=e^{-\frac{r i s}{2}}$ are selected on the right side. Moreover, put

$$
u\left(z_{1}, z_{2}\right)=\left|u_{2,2}\left(z_{1}, z_{2}\right)\right|=\cdot\left(x_{1}^{2}+\left[y_{1}+1\right]^{2}\right)^{-1}\left(x_{2}^{2}+\left[y_{2}+1\right]^{2}\right)^{-1}
$$

for $\left(z_{1}, z_{2}\right) \in \overline{\mathbb{C}_{+}^{2}}$. 
Theorem 1.2. A non-negative 2-subharmonic function $h$ on $\mathbb{C}_{+}^{2}$ has a 2-harmonic majorant if and only if

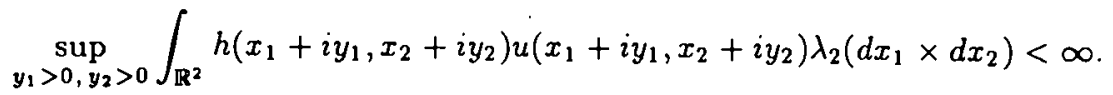

To prove this theorem we need the following lemma.

Lemma 1.3. Let $\eta$ be a non-negative function on $(0,1)^{2}$ such that the functions $r_{1} \mapsto \eta\left(r_{1}, r_{2}\right)$ for fixed $r_{2}$ and $r_{2} \mapsto \eta\left(r_{1}, r_{2}\right)$ for fixed $r_{1}$ are increasing on $(0,1)$. Furthermore, let $\zeta$ be a non-negative measurable function on $(0,1)$ with the following properties:

(i) $0<\int_{\left(0, r_{1}\right) \times\left(0, r_{2}\right)} \zeta d \lambda_{2}<\infty$ for all $\left(r_{1}, r_{2}\right) \in(0,1)^{2}$.

(ii) $\lim _{s \rightarrow 1-} \frac{\int_{(0, r s) \times(0, s)} \zeta d \lambda_{2}}{\int_{(0, s) \times(0, s)} \zeta d \lambda_{2}}=0$ and $\lim _{s \rightarrow 1-} \frac{\int_{(0, s) \times(0, r s)} \zeta d \lambda_{2}}{\int_{(0, s) \times(0, s)} \zeta d \lambda_{2}}=0$ for all $r \in(0,1)$.

Then

$$
\lim _{r_{1} \rightarrow 1-, r_{2} \rightarrow 1-} \eta\left(r_{1}, r_{2}\right)=\sup _{s \in(0,1)} \frac{\int_{(0,1)^{2}} \eta\left(t_{1}, t_{2}\right) \zeta\left(s t_{1}, s t_{2}\right) \lambda_{2}\left(d t_{1} \times d t_{2}\right)}{\int_{(0,1)^{2}} \zeta\left(s t_{1}, s t_{2}\right) \lambda_{2}\left(d t_{1} \times d t_{2}\right)}
$$

where we allow the possibility that both sides diverge to infinity.

Proof. Let

$$
\xi(s):=\frac{\int_{(0,1)^{2}} \eta\left(t_{1}, t_{2}\right) \zeta\left(s t_{1}, s t_{2}\right) \lambda_{2}\left(d t_{1} \times d t_{2}\right)}{\int_{(0,1)^{2}} \zeta\left(s t_{1}, s t_{2}\right) \lambda_{2}\left(d t_{1} \times d t_{2}\right)}
$$

for $s \in(0,1)$. The inequality

$$
\lim _{r_{1} \rightarrow 1-, r_{2} \rightarrow 1-} \eta\left(r_{1}, r_{2}\right) \geq \sup _{s \in(0,1)} \xi(s)
$$

is obvious, and if $\sup _{s \in(0,1)} \xi(s)=\infty$, then we have the reverse inequality as well. Hence it remains to show that in the case $\sup _{s \in(0,1)} \xi(s)=K<\infty$ the inequality $\eta\left(r_{1}, r_{2}\right) \leq K$ holds for all $\left(r_{1}, r_{2}\right) \in(0,1)^{2}$. For arbitrary $s, r_{1}$ and $r_{2}$ belonging to $(0,1)$, we find that

$$
\begin{aligned}
K & \geq \xi(s) \\
\geq & \frac{\int_{\left(r_{1}, 1\right) \times\left(r_{2}, 1\right)} \eta\left(t_{1}, t_{2}\right) \zeta\left(s t_{1}, s t_{2}\right) \lambda_{2}\left(d t_{1} \times d t_{2}\right)}{\int_{(0,1)^{2}} \zeta\left(s t_{1}, s t_{2}\right) \lambda_{2}\left(d t_{1} \times d t_{2}\right)} \\
\geq & \eta\left(r_{1}, r_{2}\right) \frac{\int_{\left(r_{1}, 1\right) \times\left(r_{2}, 1\right)} \zeta\left(s t_{1}, s t_{2}\right) \lambda_{2}\left(d t_{1} \times d t_{2}\right)}{\int_{(0,1)^{2}} \zeta\left(s t_{1}, s t_{2}\right) \lambda_{2}\left(d t_{1} \times d t_{2}\right)} \\
\geq & \eta\left(r_{1}, r_{2}\right)\left[1-\frac{\int_{(0,1) \times\left(0, r_{2}\right)} \zeta\left(s t_{1}, s t_{2}\right) \lambda_{2}\left(d t_{1} \times d t_{2}\right)}{\int_{(0,1)^{2}} \zeta\left(s t_{1}, s t_{2}\right) \lambda_{2}\left(d t_{1} \times d t_{2}\right)}\right. \\
& \left.-\frac{\int_{\left(0, r_{1}\right) \times(0,1)} \zeta\left(s t_{1}, s t_{2}\right) \lambda_{2}\left(d t_{1} \times d t_{2}\right)}{\int_{(0,1)^{2}} \zeta\left(s t_{1}, s t_{2}\right) \lambda_{2}\left(d t_{1} \times d t_{2}\right)}\right] .
\end{aligned}
$$

As $s$ increases to 1 , we get $K \geq \eta\left(r_{1}, r_{2}\right)$ by property (ii) 
Proof of Theorem 1.2. Assume that $h$ has a 2-harmonic majorant. Then there exist measures $\mu, \mu_{1}$ and $\mu_{2}$, and a constant $\rho$, satisfying the conclusion of Lemma 1.1 . Now, elementary estimates following [14: p. 137] give us

$$
\begin{array}{rl}
\int_{\mathbb{R}^{2}} & h\left(x_{1}+i y_{1}, x_{2}+i y_{2}\right) u\left(x_{1}+i y_{1}, x_{2}+i y_{2}\right) \lambda_{2}\left(d x_{1} \times d x_{2}\right) \\
\leq & \int_{\mathbb{R}^{4}} P\left(x_{1}-t_{1}, y_{1}\right) P\left(x_{2}-t_{2}, y_{2}\right) u\left(x_{1}+i y_{1}, x_{2}+i y_{2}\right) \mu\left(d t_{1} \times d t_{2}\right) \lambda_{2}\left(d x_{1} \times d x_{2}\right) \\
& +\frac{y_{2}}{\pi} \int_{\mathbb{R}^{3}} P\left(x_{1}-t_{1}, y_{1}\right) u\left(x_{1}+i y_{1}, x_{2}+i y_{2}\right) \mu_{1}\left(d t_{1}\right) \lambda_{2}\left(d x_{1} \times d x_{2}\right) \\
& +\frac{y_{1}}{\pi} \int_{\mathbb{R}^{3}} P\left(x_{2}-t_{2}, y_{2}\right) u\left(x_{1}+i y_{1}, x_{2}+i y_{2}\right) \mu_{2}\left(d t_{2}\right) \lambda_{2}\left(d x_{1} \times d x_{2}\right) \\
& +\rho y_{1} y_{2} \int_{\mathbb{R}^{2}} u\left(x_{1}+i y_{1}, x_{2}+i y_{2}\right) \lambda_{2}\left(d x_{1} \times d x_{2}\right) \\
\leq & \frac{2 y_{1}+1}{y_{1}+1} \cdot \frac{2 y_{2}+1}{y_{2}+1} \int_{\mathbb{R}^{2}} \frac{\mu\left(d t_{1} \times d t_{2}\right)}{\left(t_{1}^{2}+\left[2 y_{1}+1\right]^{2}\right)\left(t_{2}^{2}+\left[2 y_{2}+1\right]^{2}\right)} \\
& +\frac{2 y_{1}+1}{y_{1}+1} \cdot \frac{y_{2}}{y_{2}+1} \int_{\mathbb{R}} \frac{\mu_{1}\left(d t_{1}\right)}{\left(t_{1}^{2}+\left[2 y_{1}+1\right]^{2}\right)} \\
& +\frac{y_{1}}{y_{1}+1} \cdot \frac{2 y_{2}+1}{y_{2}+1} \int_{\mathbb{R}} \frac{\mu_{2}\left(d t_{2}\right)}{\left(t_{2}^{2}+\left[2 y_{2}+1\right]^{2}\right)} \\
& +\rho \frac{\pi y_{1}}{y_{1}+1} \cdot \frac{\pi y_{2}}{y_{2}+1} \\
\leq & 4 \int_{\mathbb{R}^{2}} \frac{\mu\left(d t_{1} \times d t_{2}\right)}{\left(t_{1}^{2}+1\right)\left(t_{2}^{2}+1\right)}+2 \int_{\mathbb{R}} \frac{\mu_{1}\left(d t_{1}\right)}{\left(t_{1}^{2}+1\right)}+2 \int_{\mathbb{R}} \frac{\mu_{2}\left(d t_{2}\right)}{\left(t_{2}^{2}+1\right)}+\rho \pi^{2} \\
< & \infty
\end{array}
$$

Conversely, assume that condition (4) holds. We have to show that $h$ or, equivalently, $h_{\alpha}$ has a 2-harmonic majorant. According to the proof of [15: Theorem 3.2.4], it suffices to verify that the function defined by

$$
\left(r_{1}, r_{2}\right) \mapsto \int_{\mathbb{Z}^{2}} h_{\alpha}\left(r_{1} e^{i \phi_{1}}, r_{2} e^{i \phi_{2}}\right) \sigma_{2}\left(d \phi_{1} \times d \phi_{2}\right)
$$

for $\left(r_{1}, r_{2}\right) \in(0,1)^{2}$ is bounded. It is enough to show that the function defined by

$$
\eta\left(r_{1}, r_{2}\right)=r_{1} r_{2} \int_{\mathbb{T}^{2}} h_{\alpha}\left(r_{1} e^{i \phi_{1}}, r_{2} e^{i \phi_{2}}\right) \sigma_{2}\left(d \phi_{1} \times d \phi_{2}\right),
$$

for $\left(r_{1}, r_{2}\right) \in(0,1)^{2}$ is bounded. Let $\zeta\left(t_{1}, t_{2}\right):=\left(1-t_{1}^{2}\right)^{-1}\left(1-t_{2}^{2}\right)^{-1}$ for $\left(t_{1}, t_{2}\right) \in(0,1)^{2}$. It is easy to see that $\zeta$ meets all of the conditions of Lemma 1.3. Hence to confirm the boundedness of $\eta$ we need only to prove that, for some constant $C$,

$$
\int_{(0,1)^{2}} \frac{\eta\left(t_{1}, t_{2}\right)}{\left(1-s^{2} t_{1}^{2}\right)\left(1-s^{2} t_{2}^{2}\right)} \sigma_{2}\left(d t_{1} \times d t_{2}\right) \leq C \cdot \int_{(0,1)^{2}} \frac{\sigma_{2}\left(d t_{1} \times d t_{2}\right)}{\left(1-s^{2} t_{1}^{2}\right)\left(1-s^{2} t_{2}^{2}\right)}
$$

holds for all $s \in(0,1)$. But this can be done by analogy to the proof in [14: pp. 138 $139]$ in the case of one variable 
Corollary 1.4. For every $p \in[1, \infty]$, the mapping $f \mapsto f_{\alpha}$ is a linear one-to-one correspondence from $\mathcal{H}^{p}\left(\mathbb{C}_{+}^{2}\right)$ onto $H^{p}\left(\mathbb{U}^{2}\right)$.

Proof. It is obvious that the mapping $f \mapsto f_{\alpha}$ is linear and one-to-one. For $p=\infty$ the entire assertion of the corollary is immediate. Thus suppose that $p \in[1, \infty)$. Then by Theorem 1.2 a function $f$ belongs to $\mathcal{H}^{p}\left(\mathbb{C}_{+}^{2}\right)$ if and only if $|f|^{p}$ has a 2 -harmonic majorant. This is equivalent for $\left|f_{\alpha}\right|^{p}$ to have a 2-harmonic majorant. But according to [15: Exercise 3.4.4/(b)], $\left|f_{\alpha}\right|^{p}$ has a 2-harmonic majorant if and only if $f_{\alpha} \in H^{p}\left(\mathbb{U}^{2}\right)$. Thus the mapping $f \rightarrow f_{\alpha}$ is onto

\section{Basic properties of $H^{p}\left(\mathbb{C}_{+}^{2}\right)$ functions}

Let $p \in[1, \infty)$. The mapping $f \mapsto u_{-\frac{2}{p},-\frac{2}{p}} f$ is a linear one-to-one correspondence from $H^{p}\left(\mathbb{C}_{+}^{2}\right)$ onto $\mathcal{H}^{p}\left(\mathbb{C}_{+}^{2}\right)$. If we combine this simple observation with Corollary 1.4 , we obtain properties of $H^{p}\left(\mathbb{C}_{+}^{2}\right)$ functions from the corresponding properties of $H^{p}\left(\mathbb{U}^{2}\right)$ functions. Among these are the behavior of non-tangential limits, logarithmic 2-subharmonicity, Cauchy's formula, and embedding into $L^{p}$.

Lemma 2.1. Let $p \in[1, \infty]$ and $z_{0} \in \mathbb{C}_{+}$. If $f \in \mathcal{H}^{p}\left(\mathbb{C}_{+}^{2}\right)$, then $f\left(\cdot, z_{0}\right)$ and $f\left(z_{0}, \cdot\right)$ belong to $\mathcal{H}^{p}\left(\mathbb{C}_{+}\right)$. If $f \in H^{p}\left(\mathbb{C}_{+}^{2}\right)$, then $f\left(\cdot, z_{0}\right)$ and $f\left(z_{0}, \cdot\right)$ belong to $H^{p}\left(\mathbb{C}_{+}\right)$.

Proof. For $p=\infty$ the claim is trivial. Let $p \in[1, \infty)$. If $f \in \mathcal{H}^{p}\left(\mathbb{C}_{+}^{2}\right)$, then the assertion follows from Corollary 1.4 and the analogous result for functions of one variable (see [14: Theorem B/p. 80]). If $f \in H^{p}\left(\mathbb{C}_{+}^{2}\right)$, then $u_{-\frac{2}{p},-\frac{2}{p}} f$ belongs to $\mathcal{H}^{p}\left(\mathbb{C}_{+}^{2}\right)$. By the case just proved, both $f\left(\cdot, z_{0}\right) u_{-\frac{2}{p},-\frac{2}{p}}\left(\cdot, z_{0}\right)$ and $f\left(z_{0}, \cdot\right) u_{-\frac{2}{p},-\frac{2}{p}}\left(z_{0}, \cdot\right)$ belong to $\mathcal{H}^{p}\left(\mathbb{C}_{+}\right)$. Hence $f\left(\cdot, z_{0}\right)$ and $f\left(z_{0}, \cdot\right)$ belong to $H^{p}\left(\mathbb{C}_{+}\right)$

In the following we use the notion of non-tangential limits of functions in $H^{p}\left(\mathbb{C}_{+}^{2}\right)$ or $\mathcal{H}^{p}\left(\mathbb{C}_{+}^{2}\right)$. For the definitions, see [17: pp. $207-208$ ] and [11: Subsection I.D.3].

Theorem 2.2. Let $p \in[1, \infty)$ and $f \in \mathcal{H}^{p}\left(\mathbb{C}_{+}^{2}\right)$. Then, for a.a. $\left(x_{1}, x_{2}\right) \in \mathbb{R}^{2}$, the non-tangential limit

$$
\tilde{f}\left(x_{1}, x_{2}\right)=\lim _{\left(z_{1}, z_{2}\right) \rightarrow\left(x_{1}, x_{2}\right)} f\left(z_{1}, z_{2}\right)
$$

exists. The function $\tilde{f}$ defined this way has the following properties:

(i) $\int_{\mathbb{R}^{2}}|\tilde{f}| d \lambda_{2}<\infty$, and for $p \in[1, \infty)$ we have $\int_{\mathbb{R}^{2}}|\tilde{f}|^{p} u d \lambda_{2}<\infty$.

(ii) $f\left(z_{1}, z_{2}\right)=P[\tilde{f}]\left(z_{1}, z_{2}\right)$ for all $\left(z_{1}, z_{2}\right) \in \mathbb{C}_{+}^{2}$.

(iii) $\int_{\mathbb{R}^{2}}|\log | \tilde{f}|| u d \lambda_{2}<\infty$ if $f$ is not the null function.

(iv) $\log \left|f\left(z_{1}, z_{2}\right)\right| \leq P[\log |\tilde{f}|]\left(z_{1}, z_{2}\right)$ for all $\left(z_{1}, z_{2}\right) \in \mathbb{C}_{+}^{2}$.

If $f \in H^{p}\left(\mathbb{C}_{+}^{2}\right)$, then $\tilde{f} \in L^{p}\left(\lambda_{2}\right)$.

Proof. If $f \in \mathcal{H}^{p}\left(\mathbb{C}_{+}^{2}\right)$, then $f_{\alpha} \in H^{p}\left(\mathbb{U}^{2}\right)$, by Corollary 1.4. According to [17: Theorem 1], the non-tangential limits of $f_{\alpha}$ exist a.e. on $\mathbb{T}^{2}$. Since $\alpha$ and $\beta$ map nontangential paths into non-tangential paths, and sets of Lebesgue measure zero into sets 
of Lebesgue measure zero, we obtain the existence of non-tangential limits of $f$ for a.a. $\left(x_{1}, x_{2}\right) \in \mathbb{R}^{2}$. The properties (i) - (iv) of $\tilde{f}$ follow from the corresponding properties of $H^{p}\left(\mathbb{U}^{2}\right)$ functions (see [15: Theorems 3.4 .2 and 3.3.5, and Execise $\left.3.4 .4 /(\mathrm{c})\right]$ ), by fractional linear transformation. Finally, Fatou's lemma shows that $\tilde{f} \in L^{p}\left(\lambda_{2}\right)$ if $f \in H^{p}\left(\mathbb{C}_{+}^{2}\right)$

The function $\tilde{f}$ defined in Theorem 2.2 is called the boundary function of $f$.

Lemma 2.3. Let $p \in[1, \infty]$ and $\tilde{f} \in L^{p}\left(\lambda_{2}\right)$. Suppose that the function $f$ given by $f\left(z_{1}, z_{2}\right)=P[\tilde{f}]\left(z_{1}, z_{2}\right)$ for $\left(z_{1}, z_{2}\right) \in \mathbb{C}_{+}^{2}$ is analytic on $\mathbb{C}_{+}^{2}$. Then $f \in H^{p}\left(\mathbb{C}_{+}^{2}\right)$, and $\tilde{f}$ is the boundary function of $f$. then

Proof. If $p=\infty$, then clearly $\left|f\left(z_{1}, z_{2}\right)\right| \leq\|\tilde{f}\|_{\infty}$ for $\left(z_{1}, z_{2}\right) \in \mathbb{C}_{+}^{2}$. If $p \in[1, \infty)$,

$$
\int_{\mathbb{R}^{2}}\left|f\left(x_{1}+i y_{1}, x_{2}+i y_{2}\right)\right|^{p} \lambda_{2}\left(d x_{1} \times d x_{2}\right) \leq\|\tilde{f}\|_{p}^{p}
$$

for $\left(y_{1}, y_{2}\right) \in(0, \infty)^{2}$, by Jensen's inequality. Thus, in either case, $f \in H^{p}\left(\mathbb{C}_{+}^{2}\right)$. Since $f$ is the Poisson integral of $\tilde{f}$, and $f$ is also the Poisson integral of its boundary function, we have by Theorem 2.2 that $\tilde{f}$ must be the boundary function of $f$

Since the correspondence $f \mapsto \tilde{f}$ is one-to-one, we will henceforth identify $f$ and $\tilde{f}$, and use the symbol $f$ for both the analytic function and its boundary function. In particular, we can identify $H^{p}\left(\mathbb{C}_{+}^{2}\right)$ and $H^{p}\left(\mathbb{U}^{2}\right)$ with certain subsets of $L^{p}\left(\lambda_{2}\right)$ and $L^{p}\left(\sigma_{2}\right)$, respectively. We can also introduce a norm on $\mathcal{H}^{p}\left(\mathbb{C}_{+}^{2}\right)(1 \leq p<\infty)$, namely

$$
\|f\|_{\mathcal{H P}^{\mathcal{P}}\left(\mathbf{C}_{+}^{2}\right)}=\int_{\mathbb{R}^{2}}|f|^{p} u d \lambda_{2}
$$

for $f \in \mathcal{H}^{p}\left(\mathbb{C}_{+}^{2}\right)$. Then we immediately obtain the following result.

Lemma 2.4. For every $p \in[1, \infty]$, the mapping

$$
f \mapsto f_{\alpha}
$$

is an isometry from $\mathcal{H}^{p}\left(\mathbb{C}_{+}^{2}\right)$ onto $H^{p}\left(\mathbb{U}^{2}\right)$. For every $p \in[1, \infty)$, the mapping

$$
f \mapsto\left(u_{\left.-\frac{2}{p},-\frac{2}{p} f\right)_{\alpha}}\right.
$$

is an isometry from $H^{p}\left(\mathbb{C}_{+}^{2}\right)$ onto $H^{p}\left(\mathbb{U}^{2}\right)$.

Corollary 2.5. For $p \in[1 ; \infty], H^{p}\left(\mathbb{C}_{+}^{2}\right)$ is a Banach space under the norm $\|\cdot\|_{p}$, and thus it can be considered as a closed subspace of $L^{p}\left(\lambda_{2}\right)$. For $p \in[1, \infty), \mathcal{H}^{p}\left(\mathbb{C}_{+}^{2}\right)$ is a Banach space under the norm $\|\cdot\|_{\mathcal{H}^{p}\left(\mathrm{C}_{+}^{2}\right)}$.

Proof. The assertion follows from Lemma 2.4 and the fact that $H^{p}\left(\mathbb{U}^{2}\right)$ is a Banach space when $p \in[1, \infty]$ (see [15: Exercise 3.4.4/(c)])

We continue now with statements concerning norm domination and norm convergence to the boundary function. 
Theorem 2.6. Let $p \in[1, \infty)$ and $f \in H^{p}\left(\mathbb{C}_{+}^{2}\right)$. Then:

(i) For arbitrary $\left(y_{1}, y_{2}\right) \in(0, \infty)^{2}$,

$$
\int_{\mathbb{R}^{2}}\left|f\left(x_{1}+i y_{1}, x_{2}+i y_{2}\right)\right|^{p} \lambda_{2}\left(d x_{1} \times d x_{2}\right) \leq\|f\|_{p}^{p} .
$$

(ii) For arbitrary $y_{0} \in(0, \infty)$, the functions

$$
\begin{aligned}
& (0, \infty) \ni y_{1} \mapsto \int_{\mathbb{R}^{2}}\left|f\left(x_{1}+i y_{1}, x_{2}+i y_{0}\right)\right|^{p} \lambda_{2}\left(d x_{1} \times d x_{2}\right) \\
& (0, \infty) \ni y_{2} \mapsto \int_{\mathbb{R}^{2}}\left|f\left(x_{1}+i y_{0}, x_{2}+i y_{2}\right)\right|^{p} \lambda_{2}\left(d x_{1} \times d x_{2}\right)
\end{aligned}
$$

are decreasing.

(iii) $\lim _{y_{1} \rightarrow 0+, y_{2} \rightarrow 0+} \int_{\mathbb{R}^{2}}\left|f\left(x_{1}+i y_{1}, x_{2}+i y_{2}\right)\right|^{p} \lambda_{2}\left(d x_{1} \times d x_{2}\right)=\|f\|_{p}^{p}$.

(iv) $\lim _{y_{1} \rightarrow 0+, y_{2} \rightarrow 0+} \int_{\mathbb{R}^{2}}\left|f\left(x_{1}+i y_{1}, x_{2}+i y_{2}\right)-f\left(x_{1}, x_{2}\right)\right|^{p} \lambda_{2}\left(d x_{1} \times d x_{2}\right)=0$.

Proof. Assertion (i) follows from Theorem 2.2/(ii) and Jensen's inequality. Assertion (ii) is a simple consequence of (i). By Fatou's lemma, we have

$$
\lim _{y_{1} \rightarrow 0+, y_{2} \rightarrow 0+} \int_{\mathbb{R}^{2}}\left|f\left(x_{1}+i y_{1}, x_{2}+i y_{2}\right)\right|^{p} \lambda_{2}\left(d x_{1} \times d x_{2}\right) \geq\|f\|_{p}^{p}
$$

Combining this with (i) we get assertion (iii). Finally, assertion (iv) can be established with the aid of (i) and (iii), as in the proof of [15: Theorem 3.4.3]

Theorem 2.7. For $f \in H^{\infty}\left(\mathbb{C}_{+}^{2}\right)$, we have $\sup _{\left(z_{1}, z_{2}\right) \in \mathbf{C}_{+}^{2}}\left|f\left(z_{1}, z_{2}\right)\right|=\|f\|_{\infty}$.

Proof. As noted before, $\left|f\left(z_{1}, z_{2}\right)\right| \leq\|f\|_{\infty}$ for all $\left(z_{1}, z_{2}\right) \in \mathbb{C}_{+}^{2}$. Now if $\left|f\left(z_{1}, z_{2}\right)\right|$ were uniformly bounded away from $\|f\|_{\infty}$ by a positive distance, then so would be a.a. the boundary values $f\left(x_{1}, x_{2}\right)$. But that would be absurd

Theorem 2.8. Let $p \in[1, \infty]$ and $f \in H^{p}\left(\mathbb{C}_{+}^{2}\right)$. Then:

(i) For a.a. $x_{1} \in \mathbb{R}$ and all $z_{2} \in \mathbb{C}_{+}$, the non-tangential limit $\lim _{z_{1} \rightarrow x_{1}} f\left(z_{1}, z_{2}\right)=$ $f_{1}\left(x_{1}, z_{2}\right)$ exists.

(ii) For a.a. $x_{1} \in \mathbb{R}$ the function $f_{1}\left(x_{1}, \cdot\right)$ belongs to $H^{p}\left(\mathbb{C}_{+}\right)$.

(iii) For a.a. $\left(x_{1}, x_{2}\right) \in \mathbb{R}^{2}$ the non-tangential limit $\lim _{z_{2} \rightarrow x_{2}} f_{1}\left(x_{1}, z_{2}\right)=f_{1}\left(x_{1}, x_{2}\right)$ exists and coincides with the boundary function of $f$.

Proof. For $f \in H^{p}\left(\mathbb{C}_{+}^{2}\right)$, we consider $g=f_{\alpha} \in H^{p}\left(\mathbb{U}^{2}\right)$, and cite the corresponding results on $H^{p}\left(\mathbb{U}^{2}\right)$ functions. Thus assertions (i) and (iii) follow immediately from [17: Theorem 2'] and [17: Theorem 5], respectively. Moreover, by [8: Theorem 2.6], for a.a. $\phi \in[-\pi, \pi)$, the function $g_{1}\left(e^{i \phi_{1}}, \cdot\right)$ belongs to $H^{p}(\mathbb{U})$, where $g_{1}$ is defined to be the non-tangential limit $g_{1}\left(e^{i \phi_{1}}, w_{2}\right)=\lim _{w_{1} \rightarrow e^{i \phi_{1}}} g\left(w_{1}, w_{2}\right)$ for a.a. $\phi_{1} \in[-\pi, \pi)$ and for all $w_{2} \in \mathbb{U}$. This implies that $g_{1}\left(e^{i \phi_{1}}, w_{2}\right)=P^{\circ}\left[g_{1}\left(e^{i \phi_{1}}, \cdot\right)\right]\left(w_{2}\right)$ for $w_{2} \in \mathbb{U}$ and for a.a. $\phi_{1} \in[-\pi, \pi)$. Returning to the upper halfplane we get $f_{1}\left(x_{1}, z_{2}\right)=P\left[f_{1}\left(x_{1}, \cdot\right)\right]\left(z_{2}\right)$ for $z_{2} \in \mathbb{C}_{+}$and for a.a. $x_{1} \in \mathbb{R}$. But from assertion (iii) and Theorem 2.2 it follows that the boundary function of $f_{1}\left(x_{1}, \cdot\right)$ belongs to $L^{p}\left(\lambda_{1}\right)$ for a.a. $x_{1}$. Hence, by Lemma 2.3, $f_{1}\left(x_{1}, \cdot\right) \in H^{p}\left(\mathbb{C}_{+}\right)$for a.a. $x_{1} \in \mathbb{R} \mathbf{~}$ 
Of course, an analogous claim holds with the first and second variables reversed. Thus the iterated non-tangential limit is a.e. independent of the order in which the separate limits are taken.

Next we turn to a version of the Cauchy integral formula on $\mathbb{C}_{+}^{2}$ and some of its consequences.

Theorem 2.9 (Cauchy Formula). Let $p \in[1, \infty)$ and $f \in H^{p}\left(\mathbb{C}_{+}^{2}\right)$. Then:

(i) For all $\left(z_{1}, z_{2}\right) \in \mathbb{C}_{+}^{2}, \frac{1}{(2 \pi i)^{2}} \int_{\mathbb{R}^{2}} \frac{f\left(t_{1}, t_{2}\right)}{\left(t_{1}-z_{1}\right)\left(t_{2}-z_{2}\right)} \lambda_{2}\left(d t_{1} \times d t_{2}\right)=f\left(z_{1}, z_{2}\right)$.

(ii) For all $\left(z_{1}, z_{2}\right) \notin \overline{\mathbb{C}_{+}^{2}}, \frac{1}{(2 \pi i)^{2}} \int_{\mathbb{R}^{2}} \frac{f\left(t_{1}, t_{2}\right)}{\left(t_{1}-z_{1}\right)\left(t_{2}-z_{2}\right)} \lambda_{2}\left(d t_{1} \times d t_{2}\right)=0$.

Proof. If $f \in H^{p}\left(\mathbb{C}_{+}^{2}\right)$, then Theorem 2.8 asserts that $f\left(\cdot, t_{2}\right) \in H^{p}\left(\mathbb{C}_{+}\right)$for a.a. $t_{2} \in \mathbb{R}$, and so the Cauchy formula for $H^{p}\left(\mathbb{C}_{+}\right)$functions gives

$$
\frac{1}{2 \pi i} \int_{\mathbb{R}} \frac{f\left(t_{1}, t_{2}\right)}{\left(t_{1}-z_{1}\right)} \lambda_{1}\left(d t_{1}\right)=f\left(z_{1}, t_{2}\right)
$$

for all $z_{1} \in \mathbb{C}_{+}$and a.a. $t_{2} \in \mathbb{R}$. But $f\left(z_{1}, \cdot\right) \in H^{p}\left(\mathbb{C}_{+}\right)$for all $z_{1} \in \mathbb{C}_{+}$, hence

$$
\frac{1}{2 \pi i} \int_{\mathbb{R}} \frac{f\left(z_{1}, t_{2}\right)}{t_{2}-z_{2}} \lambda_{2}\left(d t_{2}\right)=f\left(z_{1}, z_{2}\right)
$$

for all $z_{2} \in \mathbb{C}_{+}$. Now Fubini's theorem completes the proof of part (i). Part (ii) can be handled in a similar way

Theorem 2.10. Let $p \in[1, \infty)$ and $\tilde{f} \in L^{p}\left(\lambda_{2}\right)$. If

$$
\int_{\mathbb{R}^{2}} \frac{\tilde{f}\left(t_{1}, t_{2}\right)}{\left(t_{1}-z_{1}\right)\left(t_{2}-z_{2}\right)} \lambda_{2}\left(d t_{1} \times d t_{2}\right)=0
$$

for all $\left(z_{1}, z_{2}\right) \notin \overline{\mathbb{C}_{+}^{2}}$, then the function $f$ defined by

$$
f\left(z_{1}, z_{2}\right)=\frac{1}{(2 \pi i)^{2}} \int_{\mathbb{R}^{2}} \frac{\tilde{f}\left(t_{1}, t_{2}\right)}{\left(t_{1}-z_{1}\right)\left(t_{2}-z_{2}\right)} \lambda_{2}\left(d t_{1} \times d t_{2}\right)
$$

for $\left(z_{1}, z_{2}\right) \in \mathbb{C}_{+}^{2}$ belongs to $H^{p}\left(\mathbb{C}_{+}^{2}\right)$, and $\tilde{f}$ is the boundary function of $f$.

Proof. For $z_{2} \in \mathbb{C}_{+}$, condition (5) and Fubini's theorem imply that the function

$$
\mathbb{R} \ni t_{1} \mapsto \int_{\mathbb{R}} \frac{\tilde{f}\left(t_{1}, t_{2}\right)}{t_{2}-z_{2}} \lambda_{1}\left(d t_{2}\right)
$$

is equal to zero for a.a. $t_{1} \in \mathbb{R}$. Hence for $\left(z_{1}, z_{2}\right) \in \mathbb{C}_{+}^{2}$ we obtain

$$
\begin{aligned}
& f\left(z_{1}, z_{2}\right) \\
& =\frac{1}{(2 \pi i)^{2}} \int_{\mathbb{R}^{2}} \tilde{f}\left(t_{1}, t_{2}\right)\left[\left(t_{1}-z_{1}\right)^{-1}\left(t_{2}-z_{2}\right)^{-1}-\left(t_{1}-z_{1}^{*}\right)^{-1}\left(t_{2}-z_{2}\right)^{-1}\right] \lambda_{2}\left(d t_{1} \times d t_{2}\right) \\
& =\frac{1}{2 \pi i} \int_{\mathbb{R}} \int_{\mathbb{R}} \frac{\tilde{f}\left(t_{1}, t_{2}\right)}{t_{2}-z_{2}} \lambda_{1}\left(d t_{2}\right) P\left(x_{1}-t_{1}, y_{1}\right) \lambda_{1}\left(d t_{1}\right) \\
& =\frac{1}{2 \pi i} \int_{\mathbb{R}} \int_{\mathbb{R}} \tilde{f}\left(t_{1}, t_{2}\right)\left[\left(t_{2}-z_{2}\right)^{-1}-\left(t_{2}-z_{2}^{*}\right)^{-1}\right] \lambda_{1}\left(d t_{2}\right) P\left(x_{1}-t_{1}, y_{1}\right) \lambda_{1}\left(d t_{1}\right) \\
& =\int_{\mathbb{R}^{2}} \tilde{f}\left(t_{1}, t_{2}\right) P\left(x_{1}-t_{1}, y_{1}\right) P\left(x_{2}-t_{2}, y_{2}\right) \lambda_{2}\left(d t_{1} \times d t_{2}\right) .
\end{aligned}
$$


Now the result follows from Lemma 2.3

For $\left(s_{1}, s_{2}\right) \in \mathbb{R}^{2}$ we denote by $e_{s_{1}, s_{2}}$ the function defined by

$$
e_{s_{1}, s_{2}}\left(t_{1}, t_{2}\right)=e^{i\left(s_{1} t_{1}+s_{2} t_{2}\right)}
$$

for $\left(t_{1}, t_{2}\right) \in \mathbb{R}^{2}$. Let $p \in[1,2]$ and $f \in L^{p}\left(\lambda_{2}\right)$. By $\check{f}$ we mean the inverse Fourier transform of $f$. That is, if $p=1$, then

$$
\check{f}\left(s_{1}, s_{2}\right)=\frac{1}{2 \pi} \int_{\mathbb{R}^{2}} f e_{s_{1}, s_{2}}^{*} \lambda_{2}
$$

for $\left(s_{2}, s_{2}\right) \in \mathbb{R}^{2}$. If $p \in(1,2]$, then $\check{f}$ is the limit in $L^{q}\left(\lambda_{2}\right)$ of $\left(f \chi_{[-K, K]^{2}}\right)^{-}$, as $K \rightarrow \infty$, where $\chi_{[-K, K]^{2}}$ is the characteristic function of the set $[-K, K]^{2}$ and $q=\frac{p}{p-1}$. With that, we shall see that $H^{p}\left(\mathbb{C}_{+}^{2}\right)$ can be identified with the subspace of $L^{p}\left(\lambda_{2}\right)$ generated by frequencies in the quarterplane $\mathbb{R}_{+}^{2}$.

Theorem 2.11. If $p \in(1,2]$ and $f \in H^{p}\left(\mathbb{C}_{+}^{2}\right)$, then $f\left(s_{1}, s_{2}\right)=0$ for a.a. $\left(s_{1}, s_{2}\right) \in$ $\mathbb{R}^{2} \backslash[0, \infty)^{2}$. If $f \in H^{1}\left(\mathbb{C}_{+}^{2}\right)$, then $f\left(s_{1}, s_{2}\right)=0$ for all $\left(s_{1}, s_{2}\right) \in \mathbb{R}^{2} \backslash(0, \infty)^{2}$.

Proof . First, let $f \in H^{1}\left(\mathbb{C}_{+}^{2}\right)$. Theorem 2.8 asserts that $f\left(x_{1}, \cdot\right) \in H^{1}\left(\mathbb{C}_{+}\right)$for a.a. $x_{1} \in \mathbb{R}$. It follows that $\int_{\mathbb{R}} f\left(x_{1}, x_{2}\right) e^{-i s_{2} x_{2}} \lambda_{1}\left(d x_{2}\right)=0$ whenever $s_{2} \leq 0$, according to the known result for functions of one variable (see [11: Subsection VI.E]). Thus Fubini's theorem implies that $\bar{f}\left(s_{1}, s_{2}\right)=0$ for all $s_{2} \leq 0$ and $s_{1} \in \mathbb{R}$. Analogous arguments show that $\check{f}\left(s_{1}, s_{2}\right)=0$ for all $s_{1} \leq 0$ and $s_{2} \in \mathbb{R}$.

Next, assume that $f \in H^{p}\left(\mathbb{C}_{+}^{2}\right)$, where $p \in(1,2]$. We follow the method given in [11: Subsection VI.E]. For $n \in \mathbb{N}$, set

$$
f_{n}\left(x_{1}, x_{2}\right)=-n^{2} \frac{f\left(x_{1}, x_{2}\right)}{\left(x_{1}+i n\right)\left(x_{2}+i n\right)}
$$

for $\left(x_{1}, x_{2}\right) \in \mathbb{R}^{2}$. It is clear that $f_{n} \in H^{1}\left(\mathbb{C}_{+}^{2}\right)$. Hence $\dot{f}_{n}\left(s_{1}, s_{2}\right)=0$ for $\left(s_{1}, s_{2}\right) \in$ $\mathbb{R}^{2} \backslash[0, \infty)^{2}$. On the other hand, for some constant $c$ we have

$$
\begin{aligned}
\left\|f-f_{n}\right\|_{p}^{p} \leq & c \int_{\mathbb{R}^{2}}\left|f\left(x_{1}, x_{2}\right)-\frac{i n}{x_{1}+i n} f\left(x_{1}, x_{2}\right)\right|^{p} \lambda_{2}\left(d x_{1} \times d x_{2}\right) \\
& +c \int_{\mathbb{R}^{2}}\left|\frac{i n}{x_{1}+i n} f\left(x_{1}, x_{2}\right)-f_{n}\left(x_{1}, x_{2}\right)\right|^{p} \lambda_{2}\left(d x_{1} \times d x_{2}\right) \\
= & c \int_{\mathbb{R}^{2}}\left|\frac{x_{1}}{x_{1}+i n} f\left(x_{1}, x_{2}\right)\right|^{p} \lambda_{2}\left(d x_{1} \times d x_{2}\right) \\
& +c \int_{\mathbb{R}^{2}}\left|\frac{i n x_{2}}{\left(x_{1}+i n\right)\left(x_{2}+i n\right)} f\left(x_{1}, x_{2}\right)\right|^{p} \lambda_{2}\left(d x_{1} \times d x_{2}\right) .
\end{aligned}
$$

Now Lebesgue's dominated convergence theorem shows that $f_{n} \rightarrow f$ in $L^{p}\left(\lambda_{2}\right)$ as $n \rightarrow$ $\infty$. Thus $\tilde{f}_{n} \rightarrow \check{f}$ in $L^{q}\left(\lambda_{2}\right)$, and hence $\check{f}\left(s_{1}, s_{2}\right)=0$ for a.a. $\left(s_{1}, s_{2}\right) \in \mathbb{R}^{2} \backslash[0, \infty)^{2}$ 
Theorem 2.12. Let $p \in[1,2]$ and $f \in L^{p}\left(\lambda_{2}\right)$. If $\check{f}\left(s_{1}, s_{2}\right)=0$ for a.a. $\left(s_{1}, s_{2}\right) \in$ $\mathbb{R}^{2} \backslash[0, \infty)^{2}$, then $f$ is (the boundary function of) a function from $H^{p}\left(\mathbb{C}_{+}^{2}\right)$.

Proof. Let

$$
\begin{aligned}
g\left(z_{1}, z_{2}\right): & =\int_{\mathbb{R}^{2}} \tilde{f}\left(s_{1}, s_{2}\right) e^{i\left(z_{1} s_{1}+z_{2} s_{2}\right)} \lambda_{2}\left(d s_{1} \times d s_{2}\right) \\
& =\int_{(0, \infty)^{2}} \check{f}\left(s_{1}, s_{2}\right) e^{i\left(x_{1} s_{1}+x_{2} s_{2}\right)} e^{-\left(y_{1} s_{1}+y_{2} s_{2}\right)} \lambda_{2}\left(d s_{1} \times d s_{2}\right) \\
& =\int_{\mathbb{R}^{2}} \tilde{f}\left(s_{1}, s_{2}\right) e_{x_{1}, x_{2}}\left(s_{1}, s_{2}\right) e^{-\left(y_{1}\left|s_{1}\right|+y_{2}\left|s_{2}\right|\right)} \lambda_{2}\left(d s_{1} \times d s_{2}\right)
\end{aligned}
$$

for $\left(z_{1}, z_{2}\right) \in \mathbb{C}_{+}^{2}$. For $n \in \mathbb{N}$, set $f_{n}=f X_{[-n, n]}$. Then $\check{f}_{n} \rightarrow \check{f}$ in $L^{q}\left(\lambda_{2}\right)$ as $n \rightarrow \infty$. Hence

$$
\begin{aligned}
g\left(z_{1}, z_{2}\right)= & \lim _{n \rightarrow \infty} \int_{\mathbb{R}^{2}} \tilde{f}_{n}\left(s_{1}, s_{2}\right) e_{x_{1}, x_{2}}\left(s_{1}, s_{2}\right) e^{-\left(y_{1}\left|s_{1}\right|+y_{2}\left|s_{2}\right|\right)} \lambda_{2}\left(d s_{1} \times d s_{2}\right) \\
= & \lim _{n \rightarrow \infty} \frac{1}{(2 \pi)^{2}} \int_{[-n, n]^{2}} \int_{\mathbb{R}^{2}} e_{s_{1}, s_{2}}^{*}\left(t_{1}, t_{2}\right) e_{x_{1}, x_{2}}\left(s_{1}, s_{2}\right) e^{-\left(y_{1}\left|s_{1}\right|+y_{2}\left|s_{2}\right|\right)} \\
& \times \lambda_{2}\left(d s_{1} \times d s_{2}\right) f\left(t_{1}, t_{2}\right) \lambda_{2}\left(d t_{1} \times d t_{2}\right) \\
= & \lim _{n \rightarrow \infty} \int_{[-n, n]^{2}} P\left(x_{1}-t_{1}, y_{1}\right) P\left(x_{2}-t_{2}, y_{2}\right) f\left(t_{1}, t_{2}\right) \lambda_{2}\left(d t_{1} \times d t_{2}\right) \\
= & P[f]\left(z_{1}, z_{2}\right) .
\end{aligned}
$$

An application of Lemma 2.3 completes the proof

\section{Approximation in $L^{p}$ spaces}

Let $A\left(\mathbb{U}^{2}\right)$ be the Banach space of functions analytic in $\mathbb{U}^{2}$ and continuous in $\overline{\mathbb{U}^{2}}$ (see [15: Subsection 1.1.1]). Set $A\left(\mathbb{C}_{+}^{2}\right)=\left\{f_{\beta}: f \in A\left(\mathbb{U}^{2}\right)\right\}$. For $\left(m_{1}, m_{2}\right) \in \mathbb{Z}^{2}$, let $e_{m_{1}, m_{2}}^{\circ}$ be the function defined by

$$
e_{m_{1}, m_{2}}^{\circ}\left(\phi_{1}, \phi_{2}\right)=e^{i\left(m_{1} \phi_{1}+m_{2} \phi_{2}\right)}
$$

for $\left(\phi_{1}, \phi_{2}\right) \in[-\pi, \pi)^{2}$.

The following results concerning approximation in $H^{p}\left(\mathbb{C}_{+}^{2}\right)$ by $A\left(\mathbb{C}_{+}^{2}\right)$ functions or the exponentials $\left\{e_{s_{1}, s_{2}}\right\}$ will later underlie the development of notions of an outer function on $\mathbb{C}_{+}^{2}$.

Theorem 3.1. Let $p \in[1, \infty)$, and let $\nu$ be a finite Borel measure on $\mathbb{T}^{2}$. Then

$$
\vee_{p, \nu}\left\{e_{m_{1}, m_{2}}^{\circ}:\left(m_{1}, m_{2}\right) \in \mathbb{Z}_{+}^{2}\right\}=\vee_{p, \nu}\left\{a: a \in A\left(\mathbb{U}^{2}\right)\right\} \text {. }
$$

Proof. The inclusion

$$
\vee_{p, \nu}\left\{e_{m_{1}, m_{2}}^{\circ}:\left(m_{1}, m_{2}\right) \in \mathbb{Z}_{+}^{2}\right\} \subseteq V_{p, \nu}\left\{a: a \in A\left(\mathbb{U}^{2}\right)\right\}
$$

is obvious, and the reverse inclusion follows from the fact that each function in $A\left(\mathbb{U}^{2}\right)$ can be approximated uniformly on $\overline{\mathbb{U}^{2}}$ by polynomials in two variables 
Theorem 3.2. Let $p \in[1, \infty)$, and let $\mu$ be a finite Borel measure on $\mathbb{R}^{2}$. Then

$$
\vee_{p, \mu}\left\{e_{s_{1}, s_{2}}:\left(s_{1}, s_{2}\right) \in \mathbb{R}_{+}^{2}\right\}=\vee_{p, \mu}\left\{a: a \in A\left(\mathbb{C}_{+}^{2}\right)\right\}=\vee_{p, \mu}\left\{a u_{\frac{2}{p}, \frac{3}{p}}: a \in A\left(\mathbb{C}_{+}^{2}\right)\right\}
$$

Proof. First we prove the inclusion

$$
\vee_{p, \mu}\left\{a: a \in A\left(\mathbb{C}_{+}^{2}\right)\right\} \subseteq \vee_{p, \mu}\left\{e_{s_{1}, s_{2}}:\left(s_{1}, s_{2}\right) \in \mathbb{R}_{+}^{2}\right\}
$$

Using the corresponding fact for $A\left(\mathbb{U}^{2}\right)$ functions we obtain the inclusion

$$
\vee_{p, \mu}\left\{a: a \in A\left(\mathbb{C}_{+}^{2}\right)\right\} \subseteq \vee_{p, \mu}\left\{(-1)^{m_{1}+m_{2}} u_{m_{1}, m_{2}}\left(u_{m_{1}, m_{2}}^{*}\right)^{-1}:\left(m_{1}, m_{2}\right) \in \mathbb{Z}_{+}^{2}\right\}
$$

by fractional linear transformation. But the functions defined by

$$
u_{m_{1}, m_{2}}\left(x_{1}, x_{2}\right)\left[u_{m_{1}, m_{2}}^{*}\left(x_{1}, x_{2}\right)\right]^{-1}=\left(\frac{x_{1}-i}{x_{1}+i}\right)^{m_{1}}\left(\frac{x_{2}-i}{x_{2}+i}\right)^{m_{2}}
$$

for $\left(x_{1}, x_{2}\right) \in \mathbb{R}^{2}$ can be represented as polynomials in $u_{1,0}$ and $u_{0,1}$. Hence it is enough to show that

$$
u_{m_{1}, m_{2}} \in V_{p, \mu}\left\{e_{s_{1}, s_{2}}:\left(s_{1}, s_{2}\right) \in \mathbb{R}_{+}^{2}\right\}
$$

for all $\left(m_{1}, m_{2}\right) \in \mathbb{Z}_{+}^{2}$. According to [1: Appendix B.12], for any $m \in \mathbb{N}$ and any $\varepsilon>0$ there exists a number $K=K(m, \varepsilon)>0$ such that

$$
\left|\frac{i^{m}}{(x+i)^{m}}-\frac{1}{(m-1) !} \int_{[0, K]} t^{m-1} e^{i t(x+i)} \lambda_{1}(d t)\right|<\varepsilon
$$

for all $x \in \mathbb{R}$. We set

$$
\begin{aligned}
\rho_{m}(x) & =\frac{1}{(m-1) !} \int_{[0, K]} t^{m-1} e^{i t(x+i)} \lambda_{1}(d t) \\
\rho_{m_{1}, m_{2}}\left(x_{1}, x_{2}\right) & =\rho_{m_{1}}\left(x_{1}\right) \rho_{m_{2}}\left(x_{2}\right) \\
\rho_{m_{1}, 0}\left(x_{1}, x_{2}\right) & =\rho_{m_{1}}\left(x_{1}\right) \\
\rho_{0, m_{2}}\left(x_{1}, x_{2}\right) & =\rho_{m_{2}}\left(x_{2}\right) \\
\rho_{0,0}\left(x_{1}, x_{2}\right) & =1
\end{aligned}
$$

for $\left(x_{1}, x_{2}\right) \in \mathbb{R}^{2}$. From (7) it follows that

$$
\left.\begin{array}{l}
\int_{\mathbb{R}^{2}}\left|i^{m_{1}} u_{m_{1}, 0}-\rho_{m_{1}, 0}\right|^{p} d \mu<\varepsilon^{p} \mu\left(\mathbb{R}^{2}\right) \\
\int_{\mathbb{R}^{2}}\left|i^{m_{2}} u_{0, m_{2}}-\rho_{0, m_{2}}\right|^{p} d \mu<\varepsilon^{p} \mu\left(\mathbb{R}^{2}\right) .
\end{array}\right\}
$$

Moreover, (7) implies

$$
\left.\begin{array}{l}
\left|\rho_{m_{1}, 0}\left(x_{1}, x_{2}\right)\right| \leq 1+\varepsilon \\
\left|\rho_{0, m_{2}}\left(x_{1}, x_{2}\right)\right| \leq 1+\varepsilon
\end{array}\right\}
$$


for $\left(x_{1}, x_{2}\right) \in \mathbb{R}^{2}$. From this and the equality $\rho_{m_{1}, m_{2}}=\rho_{m_{1}, 0} \rho_{0, m_{2}}$ it is not difficult to derive the bound

$$
\int_{\mathbb{R}^{2}}\left|i^{m_{1}+m_{2}} u_{m_{1}, m_{2}}-\rho_{m_{1}, m_{2}}\right|^{p} d \mu<C \varepsilon^{p} \mu\left(\mathbb{R}^{2}\right)\left(1+[1+\varepsilon]^{p}\right)
$$

for some constant $C>0$. The relations (8) and (10), together with the equality of $u_{0,0}$ and $\rho_{0,0}$, would imply $(6)$ provided we can establish that

$$
\rho_{m_{1}, m_{2}} \in V_{p, \mu}\left\{e_{s_{1}, s_{2}}:\left(s_{1}, s_{2}\right) \in \mathbb{R}_{+}^{2}\right\}
$$

for all $\left(m_{1}, m_{2}\right) \in \mathbb{Z}_{+}^{2}$. Since $\rho_{0,0}=e_{0,0}$, this claim is trivial for $m_{1}=m_{2}=0$. Next assume that $\left(m_{1}, m_{2}\right) \in \mathbb{N}^{2}$. By [1: Appendix B.12], there exist sequences $\left\{h_{1, n}\right\}_{n \in N}$ and $\left\{h_{2, n}\right\}_{n \in N}$ of (finite) linear combinations of functions $e_{s_{1}, 0}\left(s_{1} \in \mathbb{R}_{+}\right)$and $e_{0, s_{2}}\left(s_{2} \in \mathbb{R}_{+}\right)$converging boundedly and pointwise to $\rho_{m_{1}, 0}$ and $\rho_{0, m_{2}}$, respectively. Hence the Lebesgue dominated convergence theorem implies that $\rho_{m_{1}, 0}$ and $\rho_{0, m_{2}}$ belong to $\vee_{p, \mu}\left\{e_{s_{1}, s_{2}}:\left(s_{1}, s_{2}\right) \in \mathbb{R}_{+}^{2}\right\}$. Again using $\rho_{m_{1}, m_{2}}=\rho_{m_{1}, 0} \rho_{0, m_{2}}$ and the bounds (9) we easily obtain the estimate

$$
\begin{gathered}
\int_{\mathbb{R}^{2}}\left|\rho_{m_{1}, m_{2}}\left(x_{1}, x_{2}\right)-h_{1, n}\left(x_{1}\right) h_{2, n}\left(x_{2}\right)\right|^{p} \mu\left(d x_{1} \times d x_{2}\right) \\
\leq C(1+\varepsilon)^{p} \int_{\mathbb{R}^{2}}\left|\rho_{0, m_{2}}\left(x_{1}, x_{2}\right)-h_{2, n}\left(x_{2}\right)\right|^{p} \mu\left(d x_{1} \times d x_{2}\right) \\
+C \int_{\mathbb{R}^{2}}\left|\rho_{m_{1}, 0}\left(x_{1}, x_{2}\right)-h_{1, n}\left(x_{1}\right)\right|^{p} \mu\left(d x_{1} \times d x_{2}\right)
\end{gathered}
$$

for some constant $C>0$. Now Lebesgue's theorem ensures

$$
\rho_{m_{1}, m_{2}} \in V_{p, \mu}\left\{e_{s_{1}, s_{2}}:\left(s_{1}, s_{2}\right) \in \mathbb{R}_{+}^{2}\right\}
$$

as needed. The inclusion

$$
\vee_{p, \mu}\left\{a u_{\frac{2}{p}, \frac{2}{p}}: a \in A\left(\mathbb{C}_{+}^{2}\right)\right\} \subseteq V_{p, \mu}\left\{a: a \in A\left(\mathbb{C}_{+}^{2}\right)\right\}
$$

is obvious. Finally, we prove

$$
\vee_{p, \mu}\left\{e_{s_{1}, s_{2}}:\left(s_{1}, s_{2}\right) \in \mathbb{R}_{+}^{2}\right\} \subseteq \vee_{p, \mu}\left\{a u_{\frac{2}{p}, \frac{2}{p}}: a \in A\left(\mathbb{C}_{+}^{2}\right)\right\}
$$

For this it is enough to demonstrate that if $g \in L^{q}(\mu)$ (where $q$ is the conjugate in$\operatorname{dex}$ to $p$ ) satisfies the condition $\int_{\mathbb{R}^{2}} a u_{\frac{2}{p}, \frac{2}{p}} g d \mu=0$ for all $a \in A\left(\mathbb{C}_{+}^{2}\right)$, then it follows $\int_{\mathbb{R}^{2}} e_{\boldsymbol{s}_{1}, s_{2}} g d \mu=0$ for all $\left(s_{1}, s_{2}\right) \in \mathbb{R}_{+}^{2}$. Choose a constant $\delta>\frac{2}{p}$. Then for any $\varepsilon>0$ and any $\left(s_{1}, s_{2}\right) \in \mathbb{R}_{+}^{2}$ the function defined by

$$
b_{\varepsilon}\left(z_{1}, z_{2}\right)=\frac{e_{s_{1}, s_{2}}\left(z_{1}, z_{2}\right)}{u_{\frac{2}{p}, \frac{2}{p}}\left(z_{1}, z_{2}\right)\left(i+\varepsilon z_{1}\right)^{\delta}\left(i+\varepsilon z_{2}\right)^{\delta}}
$$

for $\left(z_{1}, z_{2}\right) \in \overline{\mathbb{C}_{+}^{2}}$ belongs to $A\left(\mathbb{C}_{+}^{2}\right)$. (Here, we may choose the analytic branch of $(i+\varepsilon z)^{\delta}$ such that $i^{\delta}=e^{\frac{x i \delta}{2}}$.) Hence, by assumption,

$$
\int_{\mathbb{R}^{2}} b_{\varepsilon} u_{\frac{2}{p}, \frac{2}{p}} g d \mu=\int_{\mathbb{R}^{2}} \frac{e_{s_{1}, s_{2}}\left(x_{1}, x_{2}\right)}{\left(i+\varepsilon x_{1}\right)^{\delta}\left(i+\varepsilon x_{2}\right)^{\delta}} g\left(x_{1}, x_{2}\right) \mu\left(d x_{1} \times d x_{2}\right)=0 .
$$

If we let $\varepsilon \rightarrow 0$, the Lebesgue theorem gives $\int_{\mathbb{R}^{2}} e_{s_{1}, s_{2}} g d \mu=0$. Now (7), (11) and (12) yield the desired result 
The sets $\vee_{p, \nu}\left\{e_{m_{1}, m_{2}}^{o}:\left(m_{1}, m_{2}\right) \in Q\right\}$, where $Q$ is the second, third or fourth quadrant of $\mathbb{Z}^{2}$, have descriptions analogous to Theorem 3.1. An analogous generalization of Theorem 3.2 is possible as well. The details are straightforward and omitted.

\section{Inner and outer functions}

The canonical factorization of functions in $H^{P}(\mathbb{U})$ and $H^{P}\left(\mathbb{C}_{+}\right)$has given us a deep understanding of their structure. The factorization fails to exist, however, in the several variables picture. Still, it is fruitful to develop the notions of inner and outer functions.

A function $f$ analytic in $\mathbb{U}^{2}$ or $\mathbb{C}_{+}^{2}$ is called an inner one if

$$
\left|f\left(z_{1}, z_{2}\right)\right| \leq 1
$$

for all $\left(z_{1}, z_{2}\right) \in \mathbb{U}^{2}$ or all $\left(z_{1}, z_{2}\right) \in \mathbb{C}_{+}^{2}$ and the boundary function is of unit modulus a.e. on $\mathbb{T}^{2}$ or on $\mathbb{R}^{2}$, respectively.

Theorem 4.1. The mapping $f \mapsto f_{\alpha}$ determines a one-to-one correspondence from the set of inner functions on $\mathbb{C}_{+}^{2}$ onto the set of inner functions on $\mathbb{U}^{2}$.

Proof. The assertion follows immediately from the definition of an inner function on $\mathbb{C}_{+}^{2}$ or $\mathbb{U}^{2}$, respectively

Let $p \in[1, \infty]$. A function $f \in H^{p}\left(\mathbb{C}_{+}^{2}\right)$ or $H^{p}\left(\mathbb{U}^{2}\right)$ is an outer one if

$$
P[\log |f|]\left(z_{1}, z_{2}\right)=\log \left|f\left(z_{1}, z_{2}\right)\right|
$$

for all $\left(z_{1}, z_{2}\right) \in \mathbb{C}_{+}^{2}$ or

$$
P^{\circ}[\log |f|]\left(w_{1}, w_{2}\right)=\log \left|f\left(w_{1}, w_{2}\right)\right|
$$

for all $\left(w_{1}, w_{2}\right) \in \mathbb{U}^{2}$.

Theorem 4.2. Let $p \in[1, \infty]$ and $f \in H^{p}\left(\mathbb{C}_{+}^{2}\right)$. The following conditions are equivalent:

(i) The function $f$ is an outer one on $\mathbb{C}_{+}^{2}$.

(ii) The function $f_{\alpha}$ is an outer one on $\mathbb{U}^{2}$.

(iii) $P[\log |f|](i, i)=\log |f(i, i)|$.

Proof. The equivalence of assertions (i) and (ii) follows from the definition of outer function, with the aid of the fractional linear mappings $\alpha$ and $\beta$. Next, recall that a function $g \in H^{p}\left(\mathbb{U}^{2}\right)$ is outer if and only if $P^{\circ}[\log |g|](0,0)=\log |g(0,0)|$ (see [15: Subsection 4.4.3]). From this and the above result we get the equivalence of assertions (i) and (iii)

Of course, the point $(i, i) \in \mathbb{C}_{+}^{2}$ in condition (iii) of Theorem 4.2 can be replaced by any other point $\left(z_{1}, z_{2}\right) \in \mathbb{C}_{+}^{2}$. 
Theorem 4.3. If $p \in[1, \infty)$, the mapping $f \rightarrow\left(f u_{-\frac{2}{p},-\frac{2}{p}}\right)_{\alpha}$ is a one-to-one correspondence from the set of outer functions in $H^{P}\left(\mathbb{C}_{+}^{2}\right)$ onto the set of outer functions in $H^{p}\left(\mathbb{U}^{2}\right)$. The mapping $f \rightarrow f_{\alpha}$ is a one-to-one correspondence from the set of outer functions in $H^{\infty}\left(\mathbb{C}_{+}^{2}\right)$ onto the set of outer functions in $H^{\infty}\left(\mathbb{U}^{2}\right)$.

Proof. The second assertion is obvious. To prove the first one, it suffices to show that condition (iii) of Theorem 4.2 is equivalent to the condition

$$
P\left[\log \left|f u_{-\frac{2}{p},-\frac{2}{p}}\right|\right](i, i)=\log \left|f(i, i) u_{-\frac{2}{p},-\frac{2}{p}}(i, i)\right| .
$$

But this is clear, since $P\left[\log \left|u_{1,1}\right|\right](i, i)=\log \left|u_{1,1}(i, i)\right|$

In the $H^{p}\left(\mathbb{U}^{2}\right)$ setting other versions of the outer property have been introduced and studied. The $p=2$ case, in particular, proved useful for the prediction theory of homogeneous random fields on $\mathbb{Z}^{2}[2-4,7,12,16]$. In the present work we shall develop the corresponding ideas for functions on $\mathbb{C}_{+}^{2}$, and apply them to the prediction of homogeneous random fields on $\mathbb{R}^{2}$.

A function $f \in H^{p}\left(\mathbb{U}^{2}\right)$ is weakly outer if $f\left(\cdot, e^{i \phi_{2}}\right)$ is outer for a.a. $e^{i \phi_{2}} \in \mathbb{T}$ and $f\left(e^{i \phi_{1}}, \cdot\right)$ is outer for a.a. $e^{i \phi_{1}} \in \mathbb{T}$. Likewise, a function $f \in H^{p}\left(\mathbb{C}_{+}^{2}\right)$ is weakly outer if $f\left(\cdot, x_{2}\right)$ is outer for a.a. $x_{2} \in \mathbb{R}$ and $f\left(x_{1}, \cdot\right)$ is outer for a.a. $x_{1} \in \mathbb{R}$.

If we apply the conclusions in the proofs of Theorems 4.2 and 4.4 to the one variable case, we immediately obtain the following results.

Theorem 4.4. Let $p \in[1, \infty]$ and $f \in H^{p}\left(\mathbb{C}_{+}^{2}\right)$. The function $f$ is weakly outer if and only if the function $f_{\alpha}$ is weakly outer.

Theorem 4.5. If $p \in[1, \infty)$, the mapping $f \rightarrow\left(f u_{-\frac{2}{p},-\frac{2}{p}}\right)_{\alpha}$ is a one-to-one correspondence from the set of weakly outer functions in $H^{p}\left(\mathbb{C}_{+}^{2}\right)$ onto the set of weakly outer functions of $H^{p}\left(\mathbb{U}^{2}\right)$. The mapping $f \mapsto f_{\alpha}$ is a one-to-one correspondence from the set of weakly outer functions in $H^{\infty}\left(\mathbb{C}_{+}^{2}\right)$ onto the set of weakly outer functions in $H^{\infty}\left(\mathbb{U}^{2}\right)$.

Beurling's theorem leads to a complete characterization of outer functions in the one variable cases $H^{p}(\mathbb{U})$ and $H^{p}\left(\mathbb{C}_{+}\right)$. The immediate analogue in several variables fails under the standard definition of outer functions [3: Example 2.3], but there is a valid version for weakly outer functions in $H^{p}\left(\mathbb{U}^{2}\right)$ [3: Theorem 3.5]. We shall see that a similar characterization holds for weakly outer functions in $H^{p}\left(\mathbb{C}_{+}^{2}\right)$. Let us write

$$
\begin{aligned}
& \mathcal{M}_{1}^{p}=\left\{f \in L^{p}\left(\lambda_{2}\right): f\left(\cdot, t_{2}\right) \in H^{p}\left(\mathbb{C}_{+}\right) \text {for a.a. } t_{2} \in \mathbb{R}\right\} \\
& \mathcal{M}_{2}^{p}=\left\{f \in L^{p}\left(\lambda_{2}\right): f\left(t_{1}, \cdot\right) \in H^{p}\left(\mathbb{C}_{+}\right) \text {for a.a. } t_{1} \in \mathbb{R}\right\} .
\end{aligned}
$$

If the sequence $\left\{f_{n}\right\}_{n=1}^{\infty}$ converges in $L^{p}\left(\lambda_{2}\right)$ to $f$, then for a.a. fixed $t_{2} \in \mathbb{R}$ there is a subsequence $\left\{f_{n_{k}}\right\}_{k=1}^{\infty}$ such that $\left\{f_{n_{k}}\left(\cdot, t_{2}\right)\right\}_{k=1}^{\infty}$ converges to $f\left(\cdot, t_{2}\right)$ in $L^{p}(\lambda)$. Then, for any $z \notin \mathbb{C}_{+}$,

$$
\lim _{k \rightarrow \infty} \frac{1}{2 \pi i} \int_{\mathbb{R}} \frac{f\left(t_{1}, t_{2}\right)-f_{n_{k}}\left(t_{1}, t_{2}\right)}{t_{1}-z} \lambda\left(d t_{1}\right)=0 .
$$


It now follows from the Cauchy formula that if each $f_{n}$ belongs to $\mathcal{M}_{1}^{p}$, then so does $f$. The analogous claim holds for $\mathcal{M}_{2}^{p}$. Thus $\mathcal{M}_{1}^{p}$ and $\mathcal{M}_{2}^{p}$ are closed subsets of $L^{p}\left(\lambda_{2}\right)$; indeed, they are subspaces of $L^{p}\left(\lambda_{2}\right)$. Furthermore, in the cases $p \in[1,2]$ we have the following identifications:

$$
\begin{aligned}
& \mathcal{M}_{1}^{p}=\left\{f \in L^{p}\left(\lambda_{2}\right): \tilde{f}\left(s_{1}, s_{2}\right)=0 \text { for a.a. }\left(s_{1}, s_{2}\right) \notin \mathbb{R}_{+} \times \mathbb{R}\right\} \\
& \mathcal{M}_{2}^{p}=\left\{f \in L^{p}\left(\lambda_{2}\right): \tilde{f}\left(s_{1}, s_{2}\right)=0 \text { for a.a. }\left(s_{1}, s_{2}\right) \notin \mathbb{R} \times \mathbb{R}_{+}\right\} .
\end{aligned}
$$

This follows from Fubini's theorem when $p=1$; for $p \in(1,2]$ a straightforward limiting argument suffices.

Theorem 4.6. Let $p \in[1, \infty)$ and $f \in H^{p}\left(\mathbb{C}_{+}^{2}\right)$. The function $f$ is weakly outer if and only if

$$
V_{p}\left\{f e_{s_{1}, s_{2}}:\left(s_{1}, s_{2}\right) \in \mathbb{R}_{+} \times \mathbb{R}\right\}=\mathcal{M}_{1}^{p}
$$

and

$$
V_{p}\left\{f e_{s_{1}, s_{2}}:\left(s_{1}, s_{2}\right) \in \mathbb{R} \times \mathbb{R}_{+}\right\}=\mathcal{M}_{2}^{p} .
$$

Proof. Suppose $f \in L^{p}\left(\lambda_{2}\right)$ has the property that $f\left(\cdot, t_{2}\right)$ is outer in $H^{p}\left(\mathbb{C}_{+}\right)$for a.a. $t_{2} \in \mathbb{R}$. Let $l$ be a linear functional on $L^{p}\left(\lambda_{2}\right)$ which annihilates $\vee_{p}\left\{f e_{s_{1}, s_{2}}:\left(s_{1}, s_{2}\right) \in\right.$ $\left.\mathbb{R}_{+} \times \mathbb{R}\right\}$. There exists $\Phi \in L^{q}\left(\lambda_{2}\right)$ such that $l(g)=\int g \Phi d \lambda_{2}$ for all $g \in L^{p}\left(\lambda_{2}\right)$. Hence

$$
0=l\left(f e_{s_{1}, s_{2}}\right)=\int_{\mathbb{R}^{2}} f\left(t_{1}, t_{2}\right) e_{s_{1}, s_{2}}\left(t_{1}, t_{2}\right) \Phi\left(t_{1}, t_{2}\right) \lambda_{2}\left(d t_{1} \times d t_{2}\right)
$$

for all $\left(s_{1}, s_{2}\right) \in \mathbb{R}_{+} \times \mathbb{R}$. It follows from this and the weak outer property of $f$ that $\int_{\mathbb{R}} g(t) \Phi\left(t, t_{2}\right) \lambda(d t)=0$ for a.a. $t_{2} \in \mathbb{R}$ and all $g \in H^{p}\left(\mathbb{C}_{+}\right)$. Consequently, $l$ annihilates all of $\mathcal{M}_{1}^{p}$. This proves equation (13). Equation (14) can be proved in a similar way.

Conversely, assume that $f \in H^{p}\left(\mathbb{C}_{+}^{2}\right)$ is not weakly outer. Without loss of generality, suppose that there exists a subset $A \subseteq \mathbb{R}$ of positive Lebesgue measure such that the function $f\left(\cdot, t_{2}\right)$ fails to be outer in $H^{p}\left(\mathbb{C}_{+}\right)$for all $t_{2} \in A$. Choose any weakly outer function $h \in H^{p}\left(\mathbb{C}_{+}^{2}\right)$, and consider the quantity

$$
\varepsilon(t)=\inf \int_{\mathbb{R}}\left|h\left(t_{1}, t\right)-f\left(t_{1}, t\right) \phi\left(t_{1}\right)\right|^{p} \lambda\left(d t_{1}\right)
$$

where $\phi$ varies over finite linear combinations of the functions $e^{i s t} \quad\left(s \in \mathbb{R}_{+}\right)$. By assumption, $\varepsilon(t)$ must be positive for a.a. $t \in A$. And now define

$$
\varepsilon=\inf \int_{\mathbb{R}^{2}}\left|h\left(t_{1}, t_{2}\right)-f\left(t_{1}, t_{2}\right) \psi\left(t_{1}, t_{2}\right)\right|^{p} \lambda_{2}\left(d t_{1} \times d t_{2}\right)
$$

where $\psi$ varies over finite linear combinations of the functions $e_{s_{1}, s_{2}}\left(\left(s_{1}, s_{2}\right) \in \mathbb{R}_{+} \times \mathbb{R}\right)$. Evidently, $\varepsilon \geq \int_{\mathbb{R}} \varepsilon(t) \lambda(d t)>0$. Thus the subspace $v_{p}\left\{f e_{s_{1}, s_{2}}:\left(s_{1}, s_{2}\right) \in \mathbb{R}_{+} \times \mathbb{R}\right\}$ does not contain the function $h$, and (13) fails 
Again with Beurling's theorem in mind, we consider the following definition. Let $p \in[1, \infty)$. A function $f$ in $H^{p}\left(\mathbb{U}^{2}\right)$ is strongly outer in $H^{p}\left(\mathbb{U}^{2}\right)$ if

$$
\vee_{p}\left\{f e_{m_{1}, m_{2}}^{o}:\left(m_{1}, m_{2}\right) \in \mathbb{Z}_{+}^{2}\right\}=H^{p}\left(\mathbb{U}^{2}\right) \text {. }
$$

Similarly, a function $f$ in $H^{p}\left(\mathbb{C}_{+}^{2}\right)$ is strongly outer in $H^{p}\left(\mathbb{C}_{+}^{2}\right)$ if

$$
v_{p}\left\{f e_{s_{1}, s_{2}}:\left(s_{1}, s_{2}\right) \in \mathbb{R}_{+}^{2}\right\}=H^{p}\left(\mathbb{C}_{+}^{2}\right) \text {. }
$$

Note that the definition of strongly outer depends on $p$.

Theorem 4.7. Let $p \in[1, \infty)$ and $f \in H^{p}\left(\mathbb{C}_{+}^{2}\right)$. The function $f$ is strongly outer in $H^{p}\left(\mathbb{C}_{+}^{2}\right)$ if and only if $f_{\alpha}$ is strongly outer in $H^{p}\left(\mathbb{U}^{2}\right)$.

Proof. For $f \in H^{p}\left(\mathbb{C}_{+}^{2}\right)$ fixed, let $\mu$ be the finite Borel measure on $\mathbb{R}^{2}$ given by

$$
\mu\left(d x_{1} \times d x_{2}\right)=\left|f\left(x_{1}, x_{2}\right)\right|^{p} \lambda_{2}\left(d x_{1} \times d x_{2}\right) .
$$

Then the mapping $g \mapsto g f$ is an isometry from $H^{p}\left(\mathbb{C}_{+}^{2}\right)$ into $L^{p}(\mu)$. From Theorem 3.2 it now follows that

$$
\vee_{p}\left\{f e_{\boldsymbol{s}_{1}, \boldsymbol{s}_{2}}:\left(s_{1}, s_{2}\right) \in \mathbb{R}_{+}^{2}\right\}=\vee_{p}\left\{f a: a \in A\left(\mathbb{C}_{+}^{2}\right)\right\}=\vee_{p}\left\{f a u_{\frac{2}{p}, \frac{2}{p}}: a \in A\left(\mathbb{C}_{+}^{2}\right)\right\} .
$$

Hence $f \in H^{p}\left(\mathbb{C}_{+}^{2}\right)$ is strongly outer in $H^{p}\left(\mathbb{C}_{+}^{2}\right)$ if and only if

$$
\vee_{p}\left\{f a: a \in A\left(\mathbb{C}_{+}^{2}\right)\right\}=\vee_{p}\left\{f_{a u_{\frac{2}{p}}, \frac{2}{p}}: a \in A\left(\mathbb{C}_{+}^{2}\right)\right\}=H^{p}\left(\mathbb{C}_{+}^{2}\right)
$$

In an analogous way we see that $f_{\alpha}$ is strongly outer in $H^{p}\left(\mathbb{U}^{2}\right)$ if and only if $\vee_{p}\left\{f_{\alpha} a\right.$ : $\left.a \in A\left(\mathbb{U}^{2}\right)\right\}=H^{p}\left(\mathbb{U}^{2}\right)$. Thus $f \in H^{p}\left(\mathbb{C}_{+}^{2}\right)$ is strongly outer in $H^{p}\left(\mathbb{C}_{+}^{2}\right)$ if and only if for any $h \in H^{p}\left(\mathbb{C}_{+}^{2}\right)$ and any $\varepsilon>0$ there exists a function $a \in A\left(\mathbb{C}_{+}^{2}\right)$ such that

$$
\int_{\mathbb{R}^{2}}\left|h-f u_{\frac{2}{p}, \frac{2}{p}} a\right|^{p} d \lambda_{2}=\int_{\mathbb{R}^{2}}\left|h u_{-\frac{2}{p},-\frac{2}{p}}-f a\right|^{p} u d \lambda_{2}<\varepsilon .
$$

But this is equivalent to

$$
\int_{\mathbb{T}^{2}}\left|\left(h u_{-\frac{2}{p},-\frac{2}{p}}\right)_{\alpha}-f_{\alpha} a_{\alpha}\right|^{p} d \sigma_{2}<\varepsilon
$$

By Lemma 2.4 and the observation in the beginning of this proof, this last condition is equivalent to $f_{\alpha}$ being strongly outer in $H^{p}\left(\mathbb{U}^{2}\right)$

Theorem 4.8. Let $p \in[1, \infty)$. The mapping $f \mapsto\left(f u_{-\frac{2}{p}},-\frac{2}{p}\right)_{\alpha}$ is a one-to-one correspondence from the set of strongly outer functions in $H^{p}\left(\mathbb{C}_{+}^{2}\right)$ onto the set of strongly outer functions in $H^{p}\left(\mathbb{U}^{2}\right)$.

Proof. Again using the remark at the beginning of the proof of Theorem 4.7, we conclude that $f \in H^{p}\left(\mathbb{C}_{+}^{2}\right)$ is strongly outer in $H^{p}\left(\mathbb{C}_{+}^{2}\right)$ if and only if, for any $h \in H^{p}\left(\mathbb{C}_{+}^{2}\right)$ and any $\varepsilon>0$, there exists $a \in A\left(\mathbb{C}_{+}^{2}\right)$ such that

$$
\begin{aligned}
& \int_{\mathbb{R}^{2}}|h-f a|^{p} d \lambda_{2}=\int_{\mathbb{R}^{2}}\left|h u_{-\frac{2}{p},-\frac{2}{p}}-f u_{-\frac{2}{p},-\frac{2}{p}} a\right|^{p} u d \lambda_{2}
\end{aligned}
$$

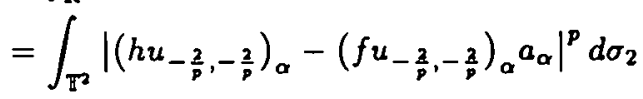

$$
\begin{aligned}
& <\varepsilon \text {. }
\end{aligned}
$$

But the last inequality is equivalent to $\left(f u_{-\frac{2}{p},-\frac{2}{p}}\right)_{\alpha}$ being strongly outer in $H^{p}\left(\mathbb{U}^{2}\right)$ 
From Theorems 4.3, 4.5 and 4.8, and the corresponding results on $\mathbb{U}^{2}$ (see [3: Proposition 2.1 and Example 2.4]), we immediately get the following fact.

Theorem 4.9. Let $p \in[1, \infty)$. There exist functions which are strongly outer in $H^{p}\left(\mathbb{C}_{+}^{2}\right)$. If a function is strongly outer in $H^{p}\left(\mathbb{C}_{+}^{2}\right)$, then it is outer. If a function is outer, then it is weakly outer. The converse implications are both false for all $p \in[1, \infty)$. Furthermore, there exist functions in $H^{\infty}\left(\mathbb{C}_{+}^{2}\right)$ which are weakly outer but not outer.

\section{Applications to prediction theory}

We shall apply the above results to the prediction theory of homogeneous random fields on $\mathbb{R}^{2}$. All notions and results will be formulated within the spectral domain of the field. Accordingly, let $\mu$ be a finite Borel measure on $\mathbb{R}^{2}$. Consider the following natural subspaces of the spectral space $L^{2}(\mu)$ :

$$
\begin{aligned}
& R_{\mu}=\vee_{2, \mu}\left\{e_{s_{1}, s_{2}}:\left(s_{1}, s_{2}\right) \in \mathbb{R}_{+} \times \mathbb{R}\right\} \\
& T_{\mu}=\vee_{2, \mu}\left\{e_{s_{1}, s_{2}}:\left(s_{1}, s_{2}\right) \in \mathbb{R} \times \mathbb{R}_{+}\right\} .
\end{aligned}
$$

The space $L^{2}(\mu)$ is said to be horizontally regular and vertically regular if

$$
\bigcap_{s_{1} \in \mathbb{R}_{+}} e_{s_{1}, 0} R_{\mu}=\{0\} \quad \text { and } \quad \bigcap_{s_{2} \in \mathbb{R}_{+}} e_{0, s_{2}} T_{\mu}=\{0\},
$$

respectively. We say that $L^{2}(\mu)$ is regular if it is both horizontally and vertically regular. By $\mathcal{P}_{R_{\mu}}$ and $\mathcal{P}_{T_{\mu}}$ we denote the orthoprojection operators of $L^{2}(\mu)$ onto $R_{\mu}$ and $T_{\mu}$, respectively. The space $L^{2}(\mu)$ has the weak commutation property if $\mathcal{P}_{R_{\mu}} \mathcal{P}_{T_{\mu}}=\mathcal{P}_{T_{\mu}} \mathcal{P}_{R_{\mu}}$. If, in addition,

$$
R_{\mu} \cap T_{\mu}=\vee_{2, \mu}\left\{e_{s_{1}, s_{2}}:\left(s_{1}, s_{2}\right) \in \mathbb{R}_{+}^{2}\right\},
$$

then $L^{2}(\mu)$ has the strong commutation property.

Let us derive some results from the existing work on random fields on $\mathbb{Z}^{2}$. We recall the corresponding definitions of regularity and the commutation properties from [2]. Let $\nu$ be a finite Borel measure on $\mathbb{T}^{2}$, viewed as the spectral measure of a homogeneous random field on $\mathbb{Z}^{2}$. Set

$$
\begin{aligned}
& R_{\nu}^{o}=\vee_{2, \nu}\left\{e_{m_{1}, m_{2}}^{o}:\left(m_{1}, m_{2}\right) \in \mathbb{Z}_{+} \times \mathbb{Z}\right\} \\
& T_{\nu}^{o}=\vee_{2, \nu}\left\{e_{m_{1}, m_{2}}^{o}:\left(m_{1}, m_{2}\right) \in \mathbb{Z} \times \mathbb{Z}_{+}\right\} .
\end{aligned}
$$

The space $L^{2}(\nu)$ is said to be horizontally regular and vetically regular if

$$
\bigcap_{m_{1} \in \mathbf{Z}_{+}} e_{m_{1}, 0}^{o} R_{\nu}^{o}=\{0\} \quad \text { and } \quad \bigcap_{m_{2} \in \mathbf{Z}_{+}} e_{0, m_{2}}^{o} T_{\nu}^{o}=\{0\}
$$

respectively. We say that $L^{2}(\nu)$ is regular if it is both horizontally and vertically regular. By $\mathcal{P}_{R_{\nu}^{\circ}}$ and $\mathcal{P}_{T_{\nu}^{\circ}}$ we denote the orthoprojection operators of $L^{2}(\nu)$ onto $R_{\nu}^{o}$ and $T_{\nu}^{o}$, respectively. 
The space $L^{2}(\nu)$ has the weak commutation property if

$$
\mathcal{P}_{R_{\nu}} \mathcal{P}_{T_{\triangleright}^{\circ}}=\mathcal{P}_{T_{\nu}} \mathcal{P}_{R_{\nu}^{\circ}}
$$

If, in addition,

$$
R_{\nu}^{o} \cap T_{\nu}^{o}=\vee_{2, \nu}\left\{e_{m_{1}, m_{2}}^{o}:\left(m_{1}, m_{2}\right) \in \mathbb{Z}_{+}^{2}\right\},
$$

then $L^{2}(\nu)$ has the strong commutation property. These commutation properties originally arose in [10] in connection with Wold type decompositions for homogeneous random fields on $\mathbb{Z}^{2}$. Further studies $[4,7,12,13,16]$ have shown that they provide a natural environment for extending some classical ideas in prediction theory.

We now turn to the situation on $\mathbb{C}_{+}^{2}$. Given a finite Borel measure $\mu$ on $\mathbb{R}^{2}$, the measure $\mu_{\alpha}$ given by

$$
\mu_{\alpha}\left(d \phi_{1} \times d \phi_{2}\right)=\mu\left(\alpha\left(d \phi_{1}\right) \times \alpha\left(d \phi_{2}\right)\right)
$$

is a finite Borel measure on $\mathbb{T}^{2}$. For a function $f \in L^{2}\left(\mu_{\alpha}\right)$, we set

$$
(V f)\left(x_{1}, x_{2}\right)=f\left(\beta\left(x_{1}\right), \beta\left(x_{2}\right)\right)
$$

for $\left(x_{1}, x_{2}\right) \in \mathbb{R}^{2}$. From the substitution rule for integrals, and from Theorems 3.1 and 3.2 we obtain the following

Theorem 5.1. The mapping $V$ is an isometry from $L^{2}\left(\mu_{\alpha}\right)$ onto $L^{2}(\mu)$. Furthermore,

$$
V\left(V_{2, \mu_{\alpha}}\left\{e_{m_{1}, m_{2}}^{o}:\left(m_{1}, m_{2}\right) \in \mathbb{Z}_{+}^{2}\right\}\right)=V_{2, \mu}\left\{e_{s_{1}, s_{2}}:\left(s_{1}, s_{2}\right) \in \mathbb{R}_{+}^{2}\right\}
$$

In a straightforward way, we get relations analogous to (15) for the other quadrants. This implies

Corollary 5.2. We have $V R_{\mu_{\alpha}}^{o}=R_{\mu}$ and $V T_{\mu_{\alpha}}^{o}=T_{\mu}$.

At last we get

Theorem 5.3. The space $L^{2}(\mu)$ has the weak or strong commutation property if and only if the space $L^{2}\left(\mu_{\alpha}\right)$ has the weak or strong commutation property, respectively.

Proof. From Corollary 5.2 we easily get that

$$
\mathcal{P}_{R_{\mu}} \mathcal{P}_{T_{\mu}}=\mathcal{P}_{T_{\mu}} \mathcal{P}_{R_{\mu}} \Longleftrightarrow \mathcal{P}_{R_{\mu_{\alpha}}^{\circ}} \mathcal{P}_{T_{\mu_{\alpha}}^{o}}=\mathcal{P}_{T_{\mu_{\alpha}}^{\circ}} \mathcal{P}_{R_{\mu_{o}}^{\circ}}
$$

Again Corollary 5.2 and equation (15) together provide that

$$
\begin{aligned}
& R_{\mu} \cap T_{\mu}=\vee_{2, \mu}\left\{e_{s_{1}, s_{2}}:\left(s_{1}, s_{2}\right) \in \mathbb{R}_{+}^{2}\right\} \\
& \quad \Longleftrightarrow R_{\mu_{\alpha}}^{o} \cap T_{\mu_{\alpha}}^{o}=\vee_{2, \mu_{\alpha}}\left\{e_{m_{1}, m_{2}}^{o}:\left(m_{1}, m_{2}\right) \in \mathbb{Z}_{+}^{2}\right\}_{-}
\end{aligned}
$$

and the assertion is proved 
Let $\mu_{1}$ and $\mu_{\alpha, 1}$ be the first marginal measures of $\mu$ and $\mu_{\alpha}$, respectively. Chiang Tse-Pei ([5: Theorems 3.2 and 3.3] and [6: Theorem 1]) showed the following. The space $L^{2}(\mu)$ is vertically regular if and only if $\mu$ is absolutely continuous with respect to $\mu_{1} \otimes \lambda_{1}$, and

$$
\int_{\mathbb{R}} \frac{\log \xi\left(x_{1}, x_{2}\right)}{1+x_{2}^{2}} \lambda_{1}\left(d x_{2}\right)>-\infty
$$

a.e. $\left[\mu_{1}\left(d x_{1}\right)\right]$, where $\xi$ is the Radon-Nikodym derivative $\left[\frac{d \mu}{d\left(\mu_{1} \otimes \lambda_{1}\right)}\right]$. The space $L^{2}\left(\mu_{\alpha}\right)$ is vertically regular if and only if $\mu_{\alpha}$ is absolutely continuous with respect to $\mu_{\alpha, 1} \otimes \sigma_{1}$, and

$$
\int_{\mathbb{T}} \log \zeta\left(\phi_{1}, \phi_{2}\right) \sigma_{1}\left(d \phi_{2}\right)>-\infty
$$

a.e. $\left[\mu_{\alpha, 1}\left(d \phi_{1}\right)\right]$, where $\zeta$ is the Radon-Nikodym derivative $\left[\frac{d \mu_{\alpha}}{d\left(\mu_{\alpha, 1} \otimes \sigma_{1}\right)}\right]$.

It is not difficult to see that $\mu$ is absolutely continuous with respect to $\mu_{1} \otimes \lambda_{1}$ if and only if $\mu_{\alpha}$ is absolutely continuous with respect to $\mu_{\alpha, 1} \otimes \sigma_{1}$, and that the relation

$$
\xi\left(x_{1}, x_{2}\right)=\frac{\zeta\left(\beta\left(x_{1}\right), \beta\left(x_{2}\right)\right)}{1+x_{2}^{2}}
$$

holds for all $\left(x_{1}, x_{2}\right) \in \mathbb{R}^{2}$. Hence $L^{2}(\mu)$ is vertically regular if and only if $L^{2}\left(\mu_{\alpha}\right)$ is vertically regular. The analogous claim is true for horizontal regularity, and thus the following is proved.

Theorem 5.4. The space $L^{2}(\mu)$ is regular if and only if the space $L^{2}\left(\mu_{\alpha}\right)$ is regular.

Now we are able to extend a fact about homogeneous random fields on $\mathbb{Z}^{2}$ to homogeneous random fields on $\mathbb{C}_{+}^{2}$. It is a characterization of the commutation properties for regular fields. On the other hand, it is also a criterion for spectral factorization, a condition which is completely understood in one variable, but which has been elusive in the several variables situation.

Theorem 5.5. Let $\mu$ be a finite Borel measure on $\mathbb{R}^{2}$. The space $L^{2}(\mu)$ is regular and weakly or strongly commutative if and only if $\mu$ is absolutely continuous with respect to $\lambda_{2}$, and $d \mu=|f|^{2} d \lambda_{2}$ for some weakly or strongly outer function $f \in H^{2}\left(\mathbb{C}_{+}^{2}\right)$, respectively.

Proof. By Theorems 5.3 and 5.4, the space $L^{2}(\mu)$ is regular and weakly or strongly commutative if and only if the space $L^{2}\left(\mu_{\alpha}\right)$ is regular and weakly or strongly commutative, respectively. But by [2: Theorem 1.1], the latter is equivalent to $\mu_{\alpha}$ being of the form $d \mu_{\alpha}=|g|^{2} d \sigma_{2}$ for some weakly or strongly outer function $g \in H^{2}\left(\mathbb{U}^{2}\right)$, respectively. It is simple to check that $\mu$ is absolutely continuous with respect to $\lambda_{2}$ if and only if $\mu_{\alpha}$ is absolutely continuous with respect to $\sigma_{2}$, and in that case the Radon-Nikodym derivative $\gamma=\left[\frac{d \mu}{d \lambda_{2}}\right]$ is given by $\gamma\left(x_{1}, x_{2}\right)=\left|g_{\beta}\left(x_{1}, x_{2}\right)\right|^{2} u\left(x_{1}, x_{2}\right)$ for $\left(x_{1}, x_{2}\right) \in \mathbb{R}^{2}$. Define $f=g_{\beta} u_{1,1}$ and note that, by Theorems 4.5 and 4.8 , the function $f$ is weakly or strongly outer in $H^{2}\left(\mathbb{C}_{+}^{2}\right)$ if and only if $g$ is weakly or strongly outer in $H^{2}\left(\mathbb{U}^{2}\right)$, respectively 
Corollary 5.6. The spectral factorization for a regular commutative random field makes possible the following version of Szego's infimum:

(i) Suppose that $L^{2}(\mu)$ is regular and weakly commutative, so that $d \mu=|f|^{2} d \lambda_{2}$ for some weakly outer function $f \in H^{2}\left(\mathbb{C}_{+}^{2}\right)$. Then, for any $\left(s_{1}, s_{2}\right) \in \mathbb{R}_{+}^{2}$,

$$
\text { inf } \begin{aligned}
\int_{\mathbb{R}^{2}} \mid 1 & +e^{i s_{1} x_{1}} h_{1}\left(x_{1}, x_{2}\right)+\left.e^{i s_{2} x_{2}} h_{2}\left(x_{1}, x_{2}\right)\right|^{2} \mu\left(d x_{1} \times d x_{2}\right) \\
& =(2 \pi)^{2} \int_{\left[0, s_{1}\right] \times\left[0, s_{2}\right]} \check{f}\left|\left(t_{1}, t_{2}\right)\right|^{2} \lambda_{2}\left(d t_{1} \times d t_{2}\right)
\end{aligned}
$$

where the infimum is taken over the conditions $h_{1} \in R_{\mu}$ and $h_{2} \in T_{\mu}$.

(ii) Suppose that $L^{2}(\mu)$ is regular and strongly commutative, so that $d \mu=|f|^{2} d \lambda_{2}$ for some strongly outer function $f \in H^{2}\left(\mathbb{C}_{+}^{2}\right)$. Then, for any $\left(s_{1}, s_{2}\right) \in \mathbb{R}_{+}^{2}$,

$$
\begin{aligned}
\inf \int_{\mathbb{R}^{2}} \mid 1 & +e^{i s_{1} x_{1}} h_{1}\left(x_{1}, x_{2}\right)+\left.e^{i s_{2} x_{2}} h_{2}\left(x_{1}, x_{2}\right)\right|^{2} \mu\left(d x_{1} \times d x_{2}\right) \\
= & (2 \pi)^{2} \int_{\left[0, s_{1}\right] \times\left[0, s_{2}\right]} \check{f}\left|\left(t_{1}, t_{2}\right)\right|^{2} \lambda_{2}\left(d t_{1} \times d t_{2}\right)
\end{aligned}
$$

where the infimum is taken over the conditions $h_{1} \in R_{\mu} \cap T_{\mu}$ and $h_{2} \in R_{\mu} \cap T_{\mu}$.

Proof. In case (i),

$$
\begin{aligned}
& \inf \int_{\mathbb{R}^{2}}\left|1+e^{i s_{1} x_{1}} h_{1}\left(x_{1}, x_{2}\right)+e^{i s_{2} x_{2}} h_{2}\left(x_{1}, x_{2}\right)\right|^{2} \mu\left(d x_{1} \times d x_{2}\right)= \\
& \inf \int_{\mathbb{R}^{2}}\left|f\left(x_{1}, x_{2}\right)+e^{i s_{1} x_{1}} h_{1}\left(x_{1}, x_{2}\right) f\left(x_{1}, x_{2}\right)+e^{i s_{2} x_{2}} h_{2}\left(x_{1}, x_{2}\right) f\left(x_{1}, x_{2}\right)\right|^{2} \lambda_{2}\left(d x_{1} \times d x_{2}\right) .
\end{aligned}
$$

Now an application of Theorem 4.6 along with Plancherel's theorem yields the claim. Case (ii) is similar

A classical result (see [9: Section 4.7]) asserts that the projection of the "future" of a stationary process onto its "past" is finite-dimensional if and only if its spectrum is absolutely continuous with a rational density function. A regular, weakly commutative field admits a similar result, with the roles of past and future assumed by subsets associated with a sort of quarterplane. Specifically, let

$$
Q_{+}=R_{\mu} \cap T_{\mu} \quad \text { and } \quad Q_{-}=Q_{+}^{*} \text {. }
$$

Corollary 5.7. Suppose that $L^{2}(\mu)$ is regular and weakly commutative, so that $d \mu=|f|^{2} d \lambda_{2}$ for some weakly outer function $f \in H^{2}\left(\mathbb{C}_{+}^{2}\right)$. Then the subspace $\mathcal{P}_{Q_{-}} Q_{+}$ of $L^{2}(\mu)$ is finite-dimensional if and only if $\left(\frac{f}{u_{1,1}}\right)_{\alpha}$ is of the form

$$
\left(\frac{f}{u_{1,1}}\right)_{\alpha}\left(w_{1}, w_{2}\right)=\frac{h\left(w_{1}, w_{2}\right)}{k_{1}\left(w_{1}\right) k_{2}\left(w_{2}\right)}
$$

for $\left(w_{1}, w_{2}\right) \in \mathbb{T}^{2}$, where $h$ is a weakly outer polynomial in $H^{2}\left(\mathbb{U}^{2}\right)$, and $k_{1}$ and $k_{2}$ are outer polynomials of one variable.

Proof. From Corollary 5.2 we get $V^{-1} Q_{+}=V^{-1}\left(R_{\mu} \cap T_{\mu}\right)=R_{\mu_{\alpha}}^{\circ} \cap T_{\mu_{\alpha}}^{o}$. Therefore, $\mathcal{P}_{Q_{-}} Q_{+}$is finite-dimensional if and only if its isomorph in $L^{2}\left(\mu_{\alpha}\right)$ is finite-dimensional. Now the assertion follows from its analog for functions on $\mathbb{U}^{2}$ [4: Theorem 3.2], applied to the measure $d \mu_{\alpha}=\left|\left(\frac{\mathcal{L}}{u_{1,1}}\right)_{\alpha}\right|^{2} d \sigma_{2}$ 
A complete characterization of weakly outer polynomials in terms of their zero sets is given in [4: Theorem 3.1].

\section{References}

[1] Akhieser, N. I.: Vorlesungen über Approximationstheorie. Berlin: Akademie-Verlag 1970.

[2] Cheng, R.: The spectral measure of a regular stationary random field with the weak or strong commutation property. Ann. Probab. 21 (1993), 1263 - 1274.

[3] Cheng, R.: Weakly and strongly outer functions on the bidisc. Michigan Math. J. 39 (1992), 99 - 109.

[4] Cheng, R. and S. Seubert: Weakly outer polynomials. Michigan Math. J. 41 (1994), 235 -245 .

[5] Chiang, T.-P.: On the linear extrapolation of a continuous homogeneous random field. Theor. Probab. Appl. 2 (1957), $58-88$.

[6] Chiang, T.-P.: On linear extrapolation of a discrete homogeneous random field (in Rus-. sian). Dokl. Akad. Nauk SSSR 112 (1957), $207-210$.

[7] Chiang, T.-P.: The prediction theory of stationary random fields. Part III: Four-fold Wold decompositions. J. Multivar. Anal. 37 (1991), 46 - 65.

[8] Davis, C. S.: Iterated limits in $N^{\bullet}\left(\mathrm{U}^{n}\right)$. Trans. Amer. Math. Soc. 178 (1973), $139-146$.

[9] Dym, H. and H. P. McKean: Gaussian Processes, Function Theory, and the Inverse Spectral Problem. New York: Academic Press 1976.

[10] Kallianpur, G. and V. Mandrekar: Nondeterministic random fields and Wold and Halmos decompositions for commuting isometries. In: Prediction Theory and Harmonic Analysis. The Pesi Masani Volume (eds.: V. Mandrekar and G. Kallianpur). Amsterdam: NorthHolland 1983, pp. $165-190$.

[11] Koosis, P.: Introduction to $H^{p}$ Spaces. With an Appendix on Wolff's Proof of the Corona Theorem. Cambridge: Univ. Press 1980.

[12] Korezlioglu, H. and Ph. Loubaton: Spectral factorization of wide sense stationary processes on $\mathbf{Z}^{2}$. J. Multivar. Anal. 19 (1986), $24-47$.

[13] Korezlioglu, H. and $\mathrm{Ph}$. Loubaton: Prediction and spectral decomposition of wide-sense stationary processes on $\mathbf{Z}^{2}$. In: Spatial Processes and Spatial Time Series Analysis. Proceedings of the 6th Franco-Belgian Meeting of Statisticians, November 1985 (ed.: F. Droesbeke). Bruxelles: Publ. Fac. Univ. Saint-Louis 1985, pp. $127-164$.

[14] Rosenblum, M. and J. Rovnyak: Hardy Classes and Operator Theory. New York et al.: Oxford Univ. Press 1985.

[15] Rudin, W.: Function Theory in Polydiscs. New York: W. A. Benjamin 1969.

[16] Soltani, A. R.: Extrapolation and moving average representation for stationary random fields and Beurling's theorem. Ann. Prob. 12 (1984), $120-132$.

[17] Zygmund, A.: On the boundary values of functions of several complex variables. Part I. Fund. Math. 36 (1949), $207-235$. 